San Jose State University

SJSU ScholarWorks

Master's Theses

Master's Theses and Graduate Research

Fall 2012

\title{
Measurement of Laser-Induced Thermo-Elastic Deformation In an Optic Using Polarization-Based Lateral Shearing Interferometry
}

Mark A. Cordier

San Jose State University

Follow this and additional works at: https://scholarworks.sjsu.edu/etd_theses

\section{Recommended Citation}

Cordier, Mark A., "Measurement of Laser-Induced Thermo-Elastic Deformation In an Optic Using Polarization-Based Lateral Shearing Interferometry" (2012). Master's Theses. 4227.

DOI: https://doi.org/10.31979/etd.ac4g-6jpr

https://scholarworks.sjsu.edu/etd_theses/4227

This Thesis is brought to you for free and open access by the Master's Theses and Graduate Research at SJSU ScholarWorks. It has been accepted for inclusion in Master's Theses by an authorized administrator of SJSU ScholarWorks. For more information, please contact scholarworks@sjsu.edu. 


\title{
MEASUREMENT OF LASER-INDUCED THERMO-ELASTIC DEFORMATION IN AN OPTIC USING POLARIZATION-BASED LATERAL SHEARING INTERFEROMETRY
}

\author{
A Thesis \\ Presented to \\ The Faculty of the Department of Physics and Astronomy \\ San José State University \\ In Partial Fulfillment \\ of the Requirements for the Degree \\ Master of Science
}

by

Mark A. Cordier

December 2012 
(C) 2012

Mark A. Cordier

ALL RIGHTS RESERVED 
The Designated Thesis Committee Approves the Thesis Titled

\title{
MEASUREMENT OF LASER-INDUCED THERMO-ELASTIC DEFORMATION IN AN OPTIC USING POLARIZATION-BASED LATERAL SHEARING INTERFEROMETRY
}

by

Mark A. Cordier

\begin{abstract}
APPROVED FOR THE DEPARTMENT OF PHYSICS AND ASTRONOMY
\end{abstract}

\section{SAN JOSÉ STATE UNIVERSITY}

December 2012
Dr. Peter Beyersdorf
Department of Physics and Astronomy
Dr. Michael Kaufman
Department of Physics and Astronomy
Dr. Kenneth Wharton
Department of Physics and Astronomy 


\begin{abstract}
MEASUREMENT OF LASER-INDUCED THERMO-ELASTIC DEFORMATION IN AN OPTIC USING POLARIZATION-BASED LATERAL SHEARING INTERFEROMETRY
\end{abstract}

by Mark A. Cordier

A polarization-based shearing interferometer is presented that is capable of measuring the thermal deformation of a mirror subject to heating from absorption of a Gaussian laser beam. The shear is generated by spatial walk-off in a birefringent crystal. By adjusting the orientation of the crystal, the components of the wavefront gradient can be independently measured to allow determination of the full wavefront vector gradate as well as reconstruction of the wavefront.

Furthermore, the monolithic nature of the birefringent crystal allows non-critical alignment of the interferometer's components. The interferogram is modulated, and a homodyne detection algorithm analyzes the modulated interferograms to extract the components of the wavefront gradient from which the wavefront is reconstructed. The thermal deformation of the laser-heated mirror was accurately observed with a sensitivity better than $\lambda / 160$. The sensitivity of this interferometer is scale invariant, and we present a method to account for the non-uniform spatial frequency response of the interferometer. 


\section{DEDICATION}

To my family and friends. 


\section{ACKNOWLEDGEMENTS}

Words can not express how grateful I am to my family. Their love, support and constant encouragement over the years has always helped me achieve what I set out to accomplish.

Thank you also to my friends at the university, whose thoughtful discussions over the years have always proven helpful. Working together long hours on homework or in the lab made all the hours of hard work seem like fun. I will miss all the absurd discussions we had in the student lounge.

A special thank you also extends to all the colleagues and friends I have made while part of the LIGO Scientific Collaboration. It has been truly amazing getting to know such a diverse and wonderful group of people.

None of this would have been possible without the fantastic collection of professors in the Department of Physics and Astronomy at San José State University. Their constant dedication to teaching and keen interest in the intellectual growth of their students has had a profound impact on my life and academic career. Thank you to each and every one of my professors. I truly could have done none of this without their support and guidance.

My profound gratitude especially goes to Dr. Peter Beyersdorf whose thoughtful advisement and patience has helped me acquire invaluable laboratory skills beyond those which lead to the publication presented here. I have never been so challenged by an instructor so patient. I consider it a privilege to have had the opportunity to work with and learn from such a dedicated mentor. 


\section{TABLE OF CONTENTS}

\section{CHAPTER}

1 BACKGROUND 1

1.1 Gravitational Waves ... . . . . . . . . . . . . . . . 1

1.2 Laser Interferometer Gravitational-Wave Observatory (LIGO) Design

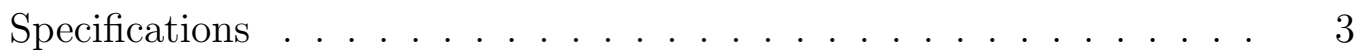

1.3 Thermal Considerations in LIGO . . . . . . . . . . . . . 4

1.3.1 The Elasto-Optic Effect . . . . . . . . . . . 6

1.3.2 The Thermo-Optic Effect . . . . . . . . . . . . . . 7

1.3.3 The Thermoelastic Effect. . . . . . . . . . . . . . 7

1.4 Sensing and Correcting Curvature . . . . . . . . . . . . . . . 8

1.5 Introduction to Shearing Interferometry . . . . . . . . . . . . . . . 9

2 THEORETICAL ANALYSIS OF LASER-INDUCED THERMAL $\begin{array}{ll}\text { DEFORMATIONS IN MIRRORS } & 10\end{array}$

2.1 Hello and Vinet's Mode of Transient Thermoelastic Deformations of Mirrors . . . . . . . . . . . . . . . . . . . . . 10

2.2 Simplified Model of Thermoelastic Deformation of Mirrors . . . . . . 12

3 SHEARING INTERFEROMETERY: THEORETICAL BACKGROUND $\begin{array}{ll}\text { AND EXPERIMENTAL DESIGN } & 16\end{array}$

3.1 Lateral Shear Interferometry . . . . . . . . . . . . . . . . 16

3.2 Shear Through Birefringence . . . . . . . . . . . . . . . . . 19

3.3 System Design . . . . . . . . . . . . . . . . . . . . 24 
3.4 Modulation . . . . . . . . . . . . . . . . . . . . . 26

3.5 Wavefront Reconstruction . . . . . . . . . . . . . . 28

3.6 Sensitivity and Resolution . . . . . . . . . . . . . . . . . 31

4 THEORETICAL BACKGROUND OF MICHELSON

INTERFEROMETRY TO CONFIRM SHEARING MEASUREMENTS 37

4.1 Michelson Interferometer Design . . . . . . . . . . . . . . 37

4.2 Spatial Frequency Modulation . . . . . . . . . . . . . . 40

4.3 Shearing Measurement Validation . . . . . . . . . . . . 46

5 SHEARING INTERFEROMETER DATA EXTRACTION AND

$\begin{array}{ll}\text { CALIBRATION } & 48\end{array}$

5.1 Experimental Details . . . . . . . . . . . . . . . . . . . 48

5.2 Calculation and Measurement of Shear . . . . . . . . . . . 51

5.3 Increasing the Dynamic Range of Shearing Interferograms _ . . . . 53

5.4 Calculation and measurement of Modulation Depth . . . . . . 57

5.5 Waverfront Reconstruction From Shearing Interferograms . . . . . . 60

5.6 Spatial Filtering and Shearing Interferometer Spatial Response . . . . 61

5.7 Shearing Measurement Confirmation _. . . . . . . . . . 62

5.8 Measured Sensitivity . . . . . . . . . . . . . . . . . . 65

5.9 Reconstruction Results . . . . . . . . . . . . . . . . 65

6 CONCLUDING REMARKS AND FUTURE DIRECTIONS 69

6.1 Applications . . . . . . . . . . . . . . . . . 69

6.2 Future Directions . . . . . . . . . . . . . . . . . . . . . . 69

6.2.1 Simultaneous Measurement of Gradient in Two Directions . . 70

6.2.2 Realtime Measurement of Thermal Deformations . . . . . 71 
6.3 Conclusion . . . . . . . . . . . . . . . . . 72

BIBLIOGRAPHY 


\section{LIST OF FIGURES}

\section{Figure}

1.1 Strain pattern induced by a gravitational wave on an imaginary ring of mass. . . . . . . . . . . . . . . . . . . .

1.2 Schematic diagram of LIGO's power-recycled Michelson interoferometer $($ not to scale). . . . . . . . . . . . . . . . 4

1.3 A correctly configured Fabry-Perot cavity (left) and a thermally deformed Fabry-Perot cavity (right). . . . . . . . . . . . .

2.1 A schematic of the thermally deformable mirror and its associated coordinate system used in the Hello and Vinet model. . . . . . . . . . 11

2.2 The calculated height of a thermal deformation across the $5 \mathrm{~mm}$ size of the imaging sensor used in this experiment. . . . . . . . . . . .

3.1 Wavefront $\phi$ interfering with a sheared version of itself, $\phi^{\prime} . \ldots 16$

3.2 Coordinates used in the paraxial approximation. . . . . . . . . . 18

3.3 A mass attached to four springs with differing spring constants $(K)$ serves as a model for an electron in an asymmetric potential well of a crystal lattice. . . . . . . . . . . . . . . . . .

3.4 Spatial walk off (or double refraction) of a laser beam transmitted through a birefringent crystal of Beta Barium Borate (BBO). . . . . 21

3.5 Schematic diagram of the shearing interferometer. . . . . . . . . 25 
3.6 Percent reduction in the measured peak surface deformation due to spatial filtering from the shearing interferometer over a spatial length scale $s . \ldots \ldots \ldots \ldots \ldots \ldots \ldots$

3.7 Theoretical bump height (blue) and the reduced height measured by the shearing interferometer (purple). . . . . . . . . . . . . 35

4.1 Schematic diagram of the Michelson interferometer used in this experiment. . . . . . . . . . . . . . . . .

4.2 An example of phase modulation where a gaussian shaped signal is used to phase modulate a cosinusoidal carrier function. . . . . . . . . 41

4.3 Schematic diagram of the tilted mirror in the Michelson interferometer which imposes a spatial carrier frequency on the measured intensity from the interferometer. . . . . . . . . . . . . . .

4.4 Fourier filtering of the modulated interference pattern from the Michelson interferometer. . . . . . . . . . . . . . .

4.5 A simulated wavefront with and without a deformation (top) and the recovered (Fourier filtered) deformation shape (bottom). . . . . . . 45

4.6 A photograph of the Michelson interferometer used in this experiment. 47

4.7 An image of the recorded interference pattern due to the undeformed test optic (left) and the thermo-elastically deformed test optic (right).

5.1 A photograph of the HeNe laser, Faraday isolator, electrooptic modulator (consisting of electrodes and a crystal of Lithium Niobate), microscope objective and pinhole used in this experiment. . . . . . . .

5.2 A photograph of the half wave plate, lens and polarizing beam splitter used in this experiment. . . . . . . . . . . . . . . 
5.3 A photograph of the Polarizing beam splitter and webcam used in this

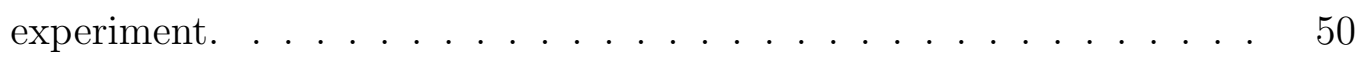

5.4 A photograph of the $\mathrm{BBO}$ in its rotating mount and the wire used to calibrate the spatial scale of the interferogram. . . . . . . . . 51

5.5 A photograph of the entire experiment and all it's components. . . . 52

5.6 An interferogram from the shearing interferometer showing horizontal

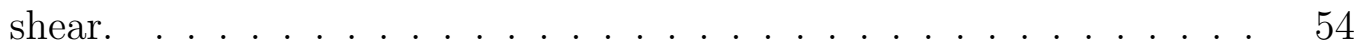

5.7 Red (upper dashed curve) and green (lower dashed curve) values for a representative pixel as a function of time together with the composite value (solid line) formed by stitching them together after appropriate scaling. . . . . . . . . . . . . . . . . . . . 55

5.8 Magnification independent sensitivity of the shearing interferometer obtained from a representative row of pixels. . . . . . . . . . 66

5.9 Height map of the thermal deformation measurement (in $\mathrm{nm}$ ) from the shearing interferometer after accounting for the spatial frequency response of the interferometer. . . . . . . . . . . . . . 67

5.10 Height map of the thermal deformation measurement (in $\mathrm{nm}$ ) from the Michelson interferometer . . . . . . . . . . . . . . . . . . 67

6.1 An alternative design to the shearing interferometer whereby two arms are used to simultaneously measure the gradient two orthogonal direc-



6.2 A second alternative design to the shearing interferometer where the modulation is achieved by rotating the $\mathrm{BBO}$ in a plane perpendicular to the optical axis allowing realtime measurement of thermal deformations. 72 


\section{CHAPTER 1}

\section{BACKGROUND}

\subsection{Gravitational Waves}

Albert Einstein revolutionized our understanding of gravitation and space-time when he published his Theory of Relativity. In General Relativity, Einstein proposed that the motion of matter is a response to distortions in four-dimensional space-time, and that perturbations in the gravitational field can be expressed as generic transverse plane wave solutions, which are free to propagate at the speed of light, just like classical electromagnetic waves. Conservation of mass/energy forbids gravitational radiation from mass monopoles, while conservation of angular momentum means that there can be no dipolar gravitational radiation. Thus the leading term in the gravitational radiation expansion is a quadrupole term. However, in contrast to electromagnetic waves, which are dipolar in nature, gravitational waves, as quadrupole waves, contract space-time in one direction while expanding space-time in an orthogonal direction, exerting a tidal strain on space-time. Thus the sources for gravitation radiation are accelerating systems where the inertia tensor for the system gives rise to a mass quadrupole moment, that is, systems whose mass distribution is neither spherically nor cylindrically symmetric $[1,15,7,28]$. It is for these reasons that anticipated sources for gravitational waves include binary inspiral of compact objects such as neutron stars and black holes, low-mass X-ray binary systems, rotational instabilities during stellar core collapse, and non-axisymmetric pulsars among others [1, 28]. 

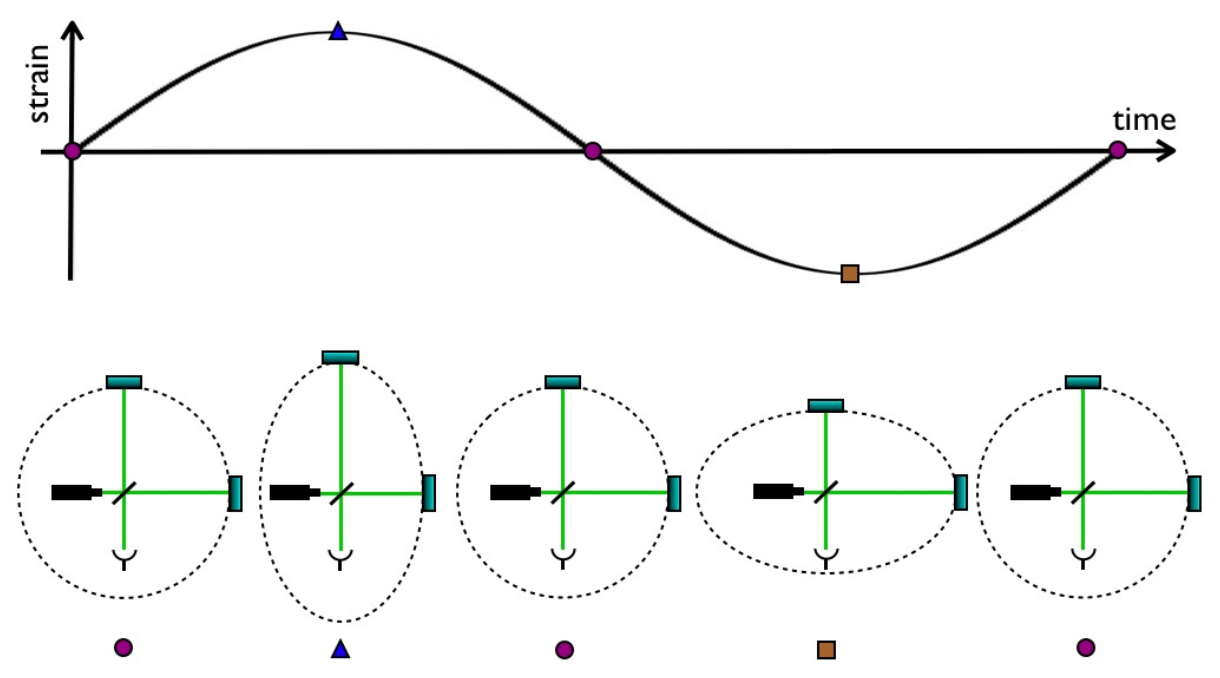

Figure 1.1: Strain pattern induced by a gravitational wave on an imaginary ring of mass.

While there is not yet any direct evidence that supports the existence of gravitational waves, the first indirect evidence came when Russle Hulse and Joseph Taylor observed a change in the period of pulses emitted by the binary pulsar PSR $1913 t+16$ over a course of 30 years, consistent with predictions made using General Relativity [27]. The Laser Interferometer Gravity-Wave Observatory (LIGO), a joint project by Caltech and Massachusetts Institute of Technology (MIT) supported by the National Science Foundation (NSF), was designed to make the first direct observation and subsequent studies of gravitational waves [1]. LIGO consists of a Michelson interferometer whose mirrors behave as test masses. The position of each mirror oscillates in response to a passing gravitational wave. Figure 1.1 illustrates the strain pattern induced by a gravitational wave propagating in a direction perpendicular to the plane of the page on an imaginary ring of mass (dashed line). When a gravitational wave interacts with the test masses of a Michelson interferometer, the relative arm lengths change, and the output fringe pattern of the interferometer varies accordingly. 


\subsection{Laser Interferometer Gravitational-Wave Observatory (LIGO) Design Specifications}

Since gravitational waves interact very weakly with matter, direct observations require extremely sensitive equipment capable of detecting changes in length of the interferometer arms on the order of $10^{-18} \mathrm{~m}$ or smaller. To put this into perspective, LIGO must be able to resolve changes in distance one thousand times smaller than the diameter of a proton [1]! While LIGO is designed to be an on-going observatory for gravitational waves, it has gone from Initial LIGO, which began data collection in 2002, and continued via small operational upgrades to Enhanced LIGO. Science runs for Initial LIGO were concluded in 2010 when site upgrades for Advanced LIGO began. Observations are scheduled to resume in 2014 [16].

In order to meet the design sensitivity, LIGO's interferometer requires careful design and monitoring of both the internal components of the interferometer as well as environmental conditions, each of which can have a direct impact on data quality. Initial LIGO employed a 10 watt continuous-wave non-planar ring oscillator (NPRO) Nd:YAG laser operating at a wavelength of $1064 \mathrm{~nm}$; this was upgraded to a $35 \mathrm{~W}$ laser for Enhanced LIGO. Advanced LIGO will use an even more powerful 200 W NPRO laser [16].

To increase the sensitivity more than would be achievable with a conventional Michelson style interferometer, LIGO was designed to be a power-recycled Michelson with Fabry-Perot cavity arms. These two modifications, shown schematically in Figure 1.2, result in a longer photon lifetime in each arm and improve the shot noise limited sensitivity, leading to better strain sensitivity. On resonance, the Fabry-Perot cavities can contain extremely high laser power, leading to thermal instabilities and increased thermal noise. The circulating power in Advanced 
LIGO's Fabry-Perot cavity arms is anticipated to reach $500 \mathrm{~kW}$ or higher [15].

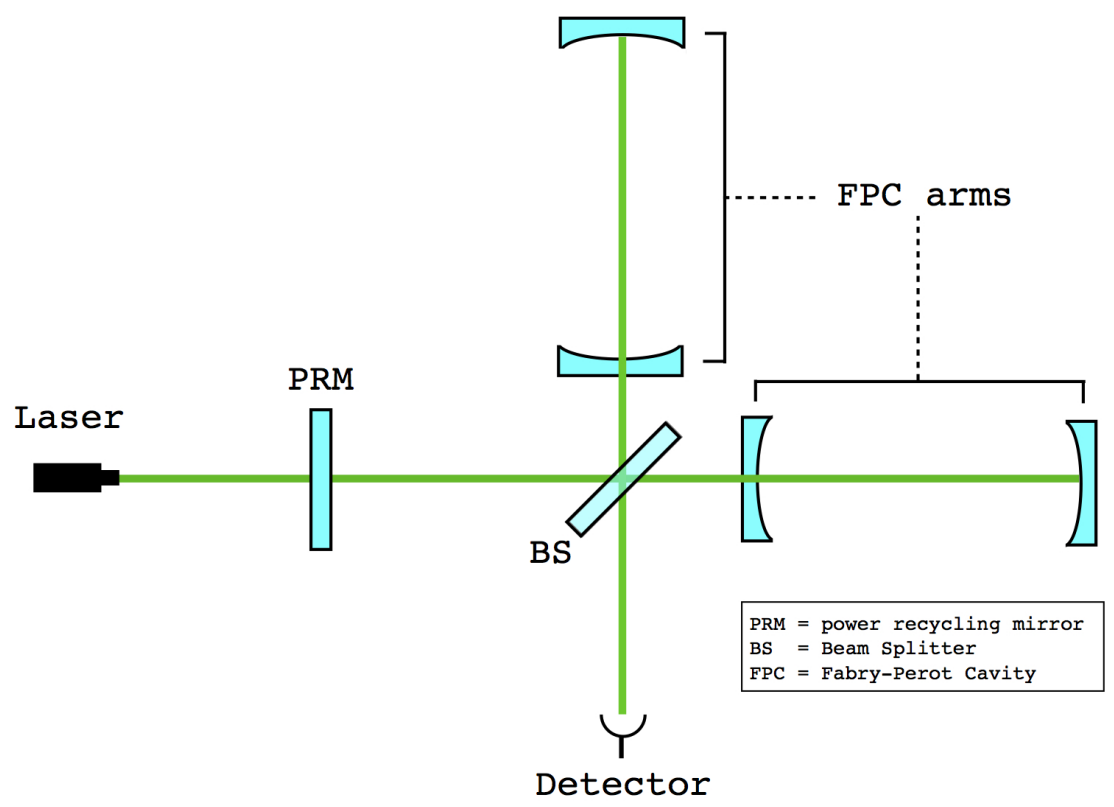

Figure 1.2: Schematic diagram of LIGO's power-recycled Michelson interoferometer (not to scale).

\subsection{Thermal Considerations in LIGO}

High power in the interferometer arms causes thermally induced deformations in the core optical components which affect the ability of the cavities to stay on resonance. The resonance conditions for a Fabry-Perot cavity require the phase front curvature of the oscillating optical field match the curvature of the cavity's mirrors at the surface of the mirror, and that the round trip phase acquired must be an integer multiple of $2 \pi$. Fulfillment of these two conditions leads to a stable mode propagating within the cavity that reproduces itself every round trip. Environmental seismic noise in the interferometer suspension, as well as thermal effects, couple into cavity length noise, both of which destabilize the resonant mode and lead to 
degraded instrument sensitivity. Figure 1.3 shows a stable cavity (left), where the phase front curvature (black dashed line) matches the curvature of the mirrors at the mirror surface, while the right figure shows thermally deformed mirrors which lead to higher cavity losses. Thermal deformations in the cavities scatter light into higher order, non-resonant modes, causing light to leave the cavity [15].
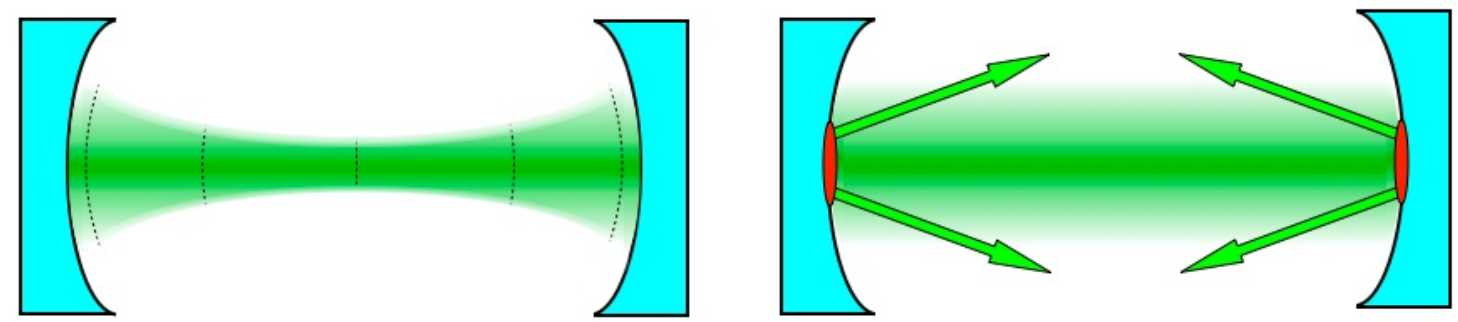

Figure 1.3: A correctly configured Fabry-Perot cavity (left) and a thermally deformed Fabry-Perot cavity (right).

Following the analysis presented by Lawrence [15], the optical path length $S_{0}$ through a material of physical thickness $L$ at a uniform temperature $T$ and with uniform index of refraction $n$ is

$$
S_{0}=n L
$$

Regardless of the type of deformation, the optical path length through a deformed optic can be expressed as

$$
S=S_{0}+\Delta S
$$

where the change in optical path length $\Delta S$ is determined from Equation 1.1 and can be attributed to either a change in the index of refraction $\Delta n$ or a change in physical thickness of the material $\Delta L$

$$
\Delta S=L \Delta n+n \Delta L
$$


We can express the change in optical path length in terms of the three primary thermal effects which can destabilize the resonant mode within the cavity: the thermo-optic effect $\Delta S_{T O}$, the elasto-optic effect $\Delta S_{E O}$ and thermoelastic deformation $\Delta S_{T E}$

$$
\Delta S_{\text {total }}=\Delta S_{E O}+\Delta S_{T O}+\Delta S_{T E}
$$

where the first two terms on the right hand side of equation 1.4 are attributable to a change in index of refraction and the last term is attributable to a change in physical thickness.

While all of these effects change the optical path length through an optic, with different relative magnitudes, shown in Table 1.1 [15], the effects are compounded by the Gaussian beam profile of the laser. The nonuniform irradience profile across the face of an optic leads to nonuniform heating, and thus nonuniform deformation.

\subsubsection{The Elasto-Optic Effect}

The elasto-optic effect (sometimes called the "photoelastic effect") occurs when a material constrained by boundary conditions is heated and expands causing a stress induced change in the index of refraction

$$
\Delta n=\frac{d n}{d T} \Delta u
$$

where $u$ is the strain in the material. The stress in the material is related to the thermal expansion $\alpha$ of the material, resulting in a polarization dependent change in optical path length. For example, an optical beam polarized in the $x$-direction sees an optical path changed by an amount

$$
\Delta S_{T O} \approx-\alpha p_{11} \int_{S} \Delta T d S
$$


where $p_{11}$ is the component of the strain-optic tensor in the direction of the beam polarzation.

\subsubsection{The Thermo-Optic Effect}

Occasionally called "thermal lensing" or "thermal blooming", the thermo-optic effect is a thermally induced change in the local index of refraction, $d n / d T$. The total difference in index between regions of different temperatures is then

$$
\Delta n_{T}=\Delta T\left(\frac{d n}{d T}\right)
$$

so the accumulated difference over the entire optical path length is

$$
\Delta S_{T O}=\int_{S}\left(\Delta n_{T}\right) d S=\frac{d n}{d T} \int_{S} \Delta T d S
$$

While the elastooptic effect is by comparison the largest of the three effects mentioned here, it effects an optical beam only as it transmits through an optic but does not affect the much higher power beam reflecting off a mirror in a Fabry-Perot cavity.

\subsubsection{The Thermoelastic Effect}

Thermoelastic deformation of an optic leads to expansion along the optical axis, changing the thickness of the of the optic. While thermoelastic deformations are smaller in magnitude than the thermo-optic effect, multiple reflections inside the Fabry-Perot cavities result in a larger net effect on the circulating optical field. The difference between the ideal curvature and deformed curvature scatters light into higher order, non-resonant modes of the Fabry-Perot cavities leading to an overall reduction in instrument sensitivity. 


\begin{tabular}{|r|l|}
\hline Thermal Loss Mechanism & Relative Strength in Silica \\
\hline Thermo-optic & 1 \\
\hline Thermal Expansion & 0.06 \\
\hline Elasto-optic & -0.01 \\
\hline
\end{tabular}

Table 1.1: Relative strengths of thermal deformation in fused silica, normalized to the magnitude of the thermo-optic effect [15].

\subsection{Sensing and Correcting Curvature}

While the LIGO cavity mirrors are engineered to be at the correct curvature when the optics are heated and the Fabry-Perot cavities are locked, the process of acquiring lock requires precise control of tip and tilt of the cavity mirrors and beam-splitter using optical levers, as well as close monitoring of the radii of

curvature of the optics. While there is an existing thermal distortion sensing system in place, it utilizes the resonant mode in the interferometer arms to provide information about the thermal deformations in the interferometer which can then be corrected. Since this method requires a resonant optical mode, it relies on critical alignment of the interferometer's core optics, and thus cannot be used to monitor the curvature of the cold cavity mirrors prior to lock.

The curvature sensor presented here can be easily incorporated in the existing LIGO geometry and has the advantage of being completely external to the vacuum chambers. This curvature sensor could allow the thermal control system to tune mirror curvature prior to lock, greatly easing the lock acquisition process. This sensor can also provide realtime feedback and monitoring of thermoelastic deformations while the interferometer is locked, thus allowing the thermal compensation system to make necessary corrections to mirror curvature in order to maintain lock. 


\subsection{Introduction to Shearing Interferometry}

Shearing interferometry is a method of common path interferometry whereby a wavefront is interfered with an image of itself that is laterally displaced by the amount of shear, $s$. The resulting interference pattern occurs only in the region of overlap and yields information about the component of the wavefront gradient in the direction of the shear. Shearing interferometers can be categorized into one of two types: separate path and near common path. In the first type, a Michelson or Mach Zehnder interferometer is typically used to generate the shear between the interfering beams, while the near common path types typically use glass wedges, etalons, or prisms to generate a shear. The near common path configurations have the advantage of mechanical stability. The typical challenge associated with using shearing interferometers is interpreting the interferograms which are a function of the wavefront gradient, not displacement [14, 34].

Measurement of the absolute wavefront profile is possible, but comparison to a reference yields a differential measurement that allows the contribution to the wavefront distortion from a particular element to be isolated. In this thesis we present a measurement of the thermal deformation of a mirror partially absorbing a Gaussian pump-beam, by measuring the difference in the wavefront with and without the pump beam illuminating the test optic. 


\section{CHAPTER 2}

\section{THEORETICAL ANALYSIS OF LASER-INDUCED THERMAL DEFORMATIONS IN MIRRORS}

\subsection{Hello and Vinet's Mode of Transient Thermoelastic Deformations of Mirrors}

In 1990, Hello and Vinet published two papers $[12,13]$ describing an analytical result for thermoelastic deformations of mirrors caused by laser heating, with intended applications in interferometeric gravity-wave detectors. In this model, the reflective coating absorbs power from the pump laser beam and subsequently heats the bulk material underneath, thermoelastically deforming the substrate.

\begin{tabular}{|c|c|c|}
\hline Variable & Parameter & Value(s) \\
\hline$\rho$ & Radial coordinate & $0 \leq \rho \leq a$ \\
\hline$z$ & Height & $-L / 2 \leq z \leq L / 2$ \\
\hline$w$ & Pump beam Gaussian radius & $0.95 \mathrm{~mm}$ \\
\hline$P$ & Pump beam power & $78.9 \mathrm{~mW}$ \\
\hline$\varepsilon$ & Fractional absorbed laser power & 0.75 \\
\hline$\kappa$ & Thermal conductivity of substrate & $1.114 \mathrm{~W} \mathrm{~m}^{-1} \mathrm{~K}$ \\
\hline$\sigma_{c}$ & Emissivity corrected Boltzmann constant & $2.8 \times 10^{-8} \mathrm{~W} \mathrm{~m}^{-2} \mathrm{~K}^{-4}$ \\
\hline$\nu$ & Poisson's Ratio & 0.206 \\
\hline$\alpha$ & Thermal expansion coefficient & $7.1 \times 10^{-6} \mathrm{~W} \mathrm{~m}^{-1} \mathrm{~K}$ \\
\hline
\end{tabular}

Table 2.1: Parameters used in the Hello \& Vinet model and Lu et al..model of thermal deformations of mirrors.

The pump laser of power $P$ has a radial intensity profile described by

$$
I(\rho)=\frac{2 P}{\pi w^{2}} e^{\left(-2 \rho^{2} / w^{2}\right)}
$$


where $w$ is the pump laser's waist, which is the point on the Gaussian radius where the intensity drops by a factor of $1 / e^{2}$. Table 2.1 contains a list of parameters and relevant values used in this analysis. The mirror is restricted to a radius of $a$ and a length $L$, whose faces are located at $z=L / 2$ and $z=-L / 2$, and the coating is assumed to be on the $z=-L / 2$ face, thus we will treat the heat source to be at this location, as shown in Figure 2.1.

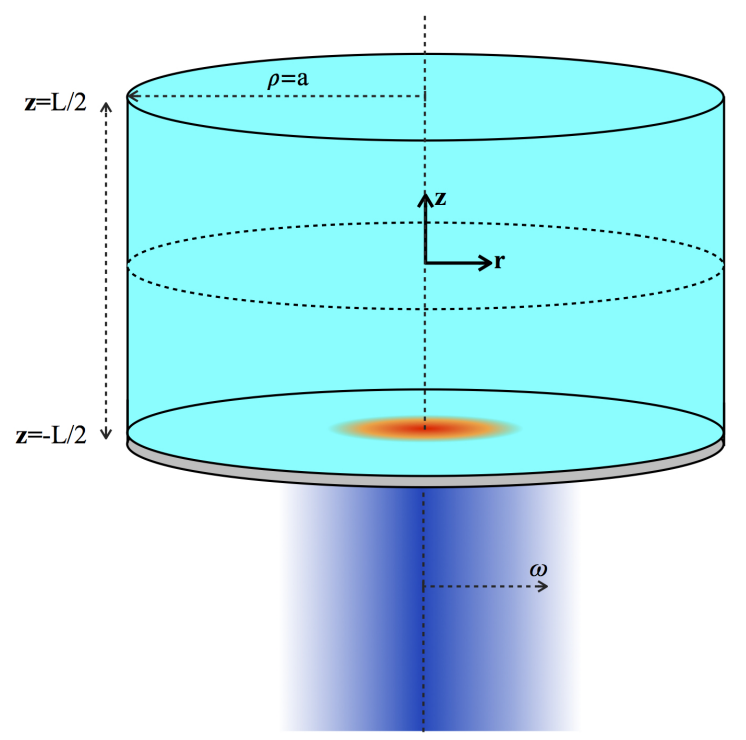

Figure 2.1: A schematic of the thermally deformable mirror and its associated coordinate system used in the Hello and Vinet model.

In the steady state with no heat generated by the substrate and the substrate allowed to cool via both radiation and conduction, the temperature is is described using the Laplace equation

$$
\begin{aligned}
\frac{\partial^{2} T}{\partial z^{2}} & =-\frac{1}{r} \partial_{r}\left(r \frac{\partial T}{\partial r}\right) \\
\nabla^{2} T(\rho, z) & =0
\end{aligned}
$$

where $T$ is the difference between the internal and external temperature of the optic, $\rho$ is the radial coordinate and $z$ is the height. The Neumann boundary 
conditions placed on the optic at the radial boundary, and the top and bottom faces of the optic are

$$
\begin{aligned}
\frac{\partial T(a, z)}{\partial z} & =\left(\frac{-4 \sigma_{c} T_{e x t}^{3}}{\kappa}\right) T(a, z) \\
\frac{\partial T(\rho,+L / 2)}{\partial z} & =\left(\frac{-4 \sigma_{c} T_{e x t}^{3}}{\kappa}\right) T(\rho,+L / 2) . \\
\frac{\partial T(\rho,-L / 2)}{\partial z} & =\left(\frac{4 \sigma_{c} T_{e x t}^{3}}{\kappa}\right) T(\rho,-L / 2)-\frac{\varepsilon}{\kappa} I(\rho) \\
& =\left(\frac{4 \sigma_{c} T_{e x t}^{3}}{\kappa}\right) T(\rho,-L / 2)-\left(\frac{\alpha}{\kappa} \frac{2 P}{\pi w^{2}}\right) e^{\left(-2 \rho^{2} / w^{2}\right)} .
\end{aligned}
$$

The exact solution to the Laplace equation subject to these boundary conditions is expressed as a Dini series and provides a temperature field which is then used to calculate surface deformation.

\subsection{Simplified Model of Thermoelastic Deformation of Mirrors}

While Hello and Vinet provided an exact result which describes the thermoelastic deformation of mirrors, a simplified analysis provided by Lu et al. [17] is more convenient for many laboratory and design applications. This model differs from that presented by Hello and Vinet in that it approximates a solution to a mirror of finite dimension by considering a half-infinite mirror instead. The relaxed constraint derives from the fact that the intensity of the pump laser becomes insignificant at radii greater than twice the beam waist $\rho \geq 2 w$, and is thus valid for mirrors whose radius $a>2 w$ and whose thickness $L>2 w$, a constraint easily met in most laboratory applications.

This analysis uses the dimensionless parameters $\bar{\rho}=\rho / w, \bar{z}=z / w, \bar{T}=T / T_{c}$, and $\bar{l}_{t h}=l_{t h} / w$ where

$$
T_{c}=\frac{2 \varepsilon P}{\pi w \kappa}
$$


is a characteristic temperature and $l_{t h}$ is a characteristic length

$$
l_{t h}=\frac{\kappa}{4 \sigma_{c} T_{e x t}^{3}}
$$

As before, the heat field is determined from the Laplace equation in terms of the dimensionless parameters as

$$
\begin{aligned}
& 0=\nabla^{2} \bar{T}(\bar{\rho}, \bar{z}) \\
& 0=\frac{\partial^{2} \bar{T}(\bar{\rho}, \bar{z})}{\partial \bar{\rho}^{2}}+\frac{1}{\bar{\rho}} \frac{\partial T(\bar{\rho}, \bar{z})}{\partial \bar{\rho}}+\frac{\partial^{2} \bar{T}(\bar{\rho}, \bar{z})}{\partial \bar{z}^{2}} .
\end{aligned}
$$

The boundary conditions on the temperature at $\rho=\infty, z=\infty$ and at the coating surface $z=-h / 2$ are

$$
\begin{aligned}
\bar{T}(\bar{\rho}=\infty, \bar{z}) & =0 \\
\bar{T}(\bar{\rho}, \bar{z}=\infty) & =0 \\
-\frac{\partial \bar{T}(\bar{\rho},-\bar{L} / 2)}{\partial \bar{z}}+\frac{1}{l_{t h}} \bar{T}(\bar{\rho},-\bar{L} / 2) & =e^{-2 \bar{\rho}^{2}} .
\end{aligned}
$$

The solutions to equation 2.10, which approach zero at infinity as required by the first two boundary conditions (Equations 2.11 and 2.12), have the form $e^{-q z} J_{0}(q \rho)$, where $q$ is a dummy variable and $J_{0}$ is a Bessel Function of the first kind. The solution which satisfies the third boundary condition (Equation 2.13) is then a superposition of the solutions which satisfy the previous two boundary conditions at the surface $z=-\bar{L} / 2$

$$
\bar{T}(\bar{\rho}, \bar{z})=\int_{0}^{\infty} A(q) e^{-(\bar{z}+\bar{L} / 2) q} J_{0}(q \bar{\rho}) d q
$$

where the amplitude $A$ of the $q^{\text {th }}$ solution is given by

$$
A(q)=\frac{1}{4} \frac{e^{-q^{2} / 8}}{\left(1+1 / q \bar{l}_{t h}\right)} .
$$


Thus, the temperature field which satisfies all of the necessary boundary conditions is given by

$$
\bar{T}(\bar{\rho}, \bar{z})=\frac{1}{4} \int_{0}^{\infty} \frac{e^{-q^{2} / 8} e^{-(\bar{z}+\bar{L} / 2) q} J_{0}(q \bar{\rho})}{\left(1+1 / q \bar{l}_{t h}\right)} d q
$$

and is then used to calculate the thermal deformation. Since we are particularly concerned with the height of the deformation, we will consider only the axial component of the surface deformation along the optical axis

$$
u_{z}(\rho, z)=-\bar{u}_{c} \int_{0}^{\bar{\rho}} \frac{d \bar{\rho}^{\prime}}{\bar{\rho}} \int_{0}^{\bar{\rho}^{\prime}} \frac{\partial \bar{T}\left(\bar{\rho}^{\prime \prime},-\bar{L} / 2\right)}{\partial \bar{z}} \rho^{\prime \prime} d \rho^{\prime \prime}
$$

where

$$
\bar{u}_{c}=\frac{2 \alpha \varepsilon P}{\pi \kappa}(1+\nu)
$$

is a characteristic displacement depending only on the illumination of the pump laser power $P$ and material properties summarized in Table 2.1 for our mirror's BK7 glass substrate.

With the radius and thickness of the mirror used in this experiment being several times greater than the Gaussian radius of the pump beam, the size and shape of the thermoelastic deformation is well approximated by the analytical solution (Equation 2.19) obtained for the thermoelastic deformation in a half-infinite mirror. The resulting shape of the deformation has been worked out in [17] giving an axial surface displacement of

$$
u_{z}(\rho)=\frac{u_{c}}{8}\left[E_{1}\left(\frac{2 \rho^{2}}{w^{2}}\right)+\ln \left(\frac{2 r^{2}}{w^{2}}\right)+\gamma\right]
$$

where $\gamma$ is Euler's constant and $E_{1}$ is the exponential integral function given in [2].

Figure 2.2 shows the anticipated height of the surface deformation of the mirror across a $5 \mathrm{~mm}$ imaging array, identical to that used in our experiment, given 
the parameters in Table 2.1 . The height of the deformation is measured down from the peak of the deformation since this model assumes a mirror of infinite spatial extent resulting in an infinite deformation height between the center of the mirror and the edge (at infinite radial distance).

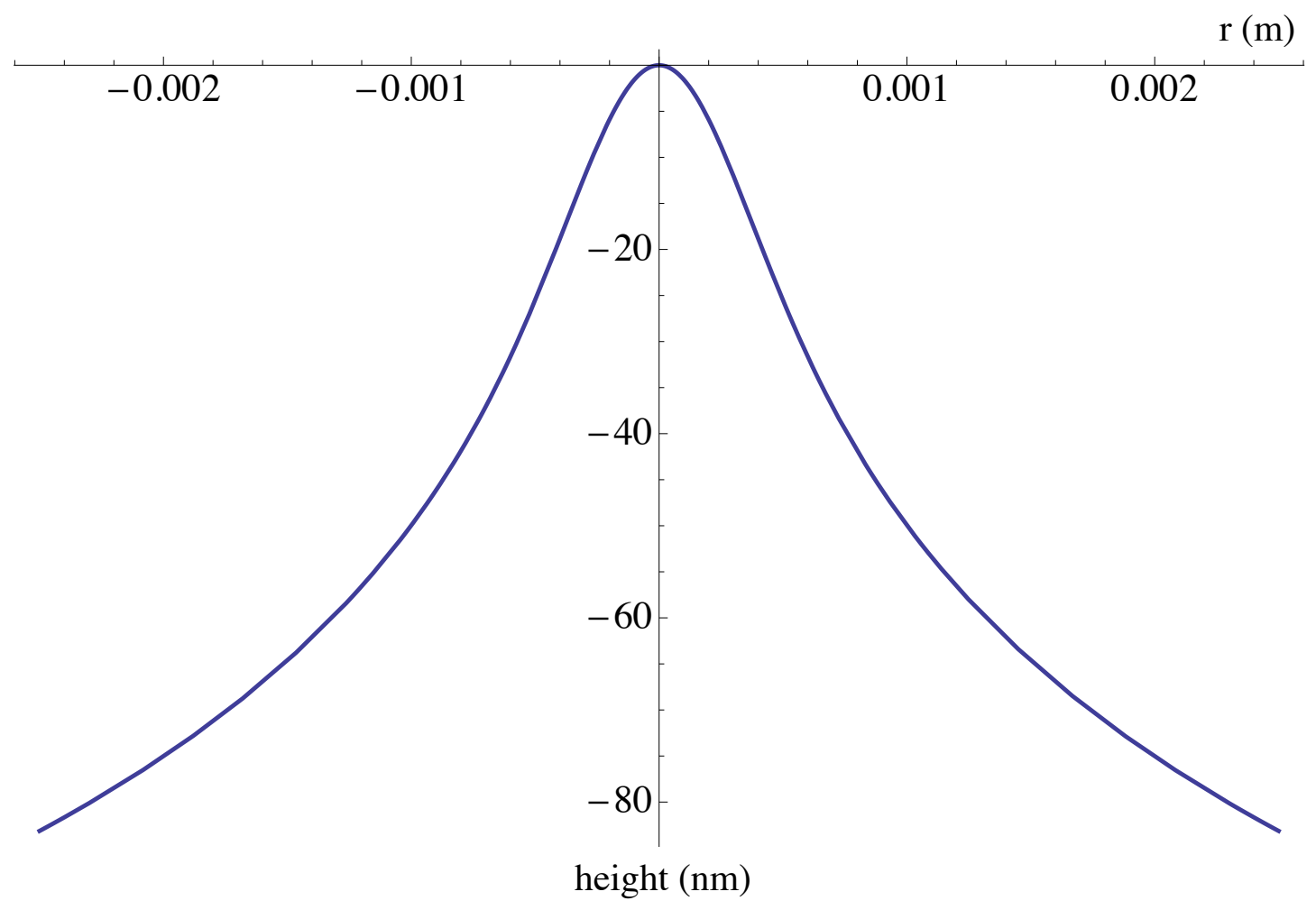

Figure 2.2: The calculated height of a thermal deformation across the $5 \mathrm{~mm}$ size of the imaging sensor used in this experiment. 


\section{CHAPTER 3}

\section{SHEARING INTERFEROMETRY: THEORETICAL BACKGROUND AND EXPERIMENTAL DESIGN}

\subsection{Lateral Shear Interferometry}

The interference pattern generated by a shearing interferometer is the result of the lateral displacement of one wavefront relative to another and contains information about the gradient of the wavefront. While rigorous analysis requires the wavefront be nearly flat, the curvature of wavefront $\phi$ in Figure 3.1 is exaggerated for pedagogical purposes. This serves to illustrate that points $A^{\prime}$ and $B$ have phases related by the gradient of the wavefront.

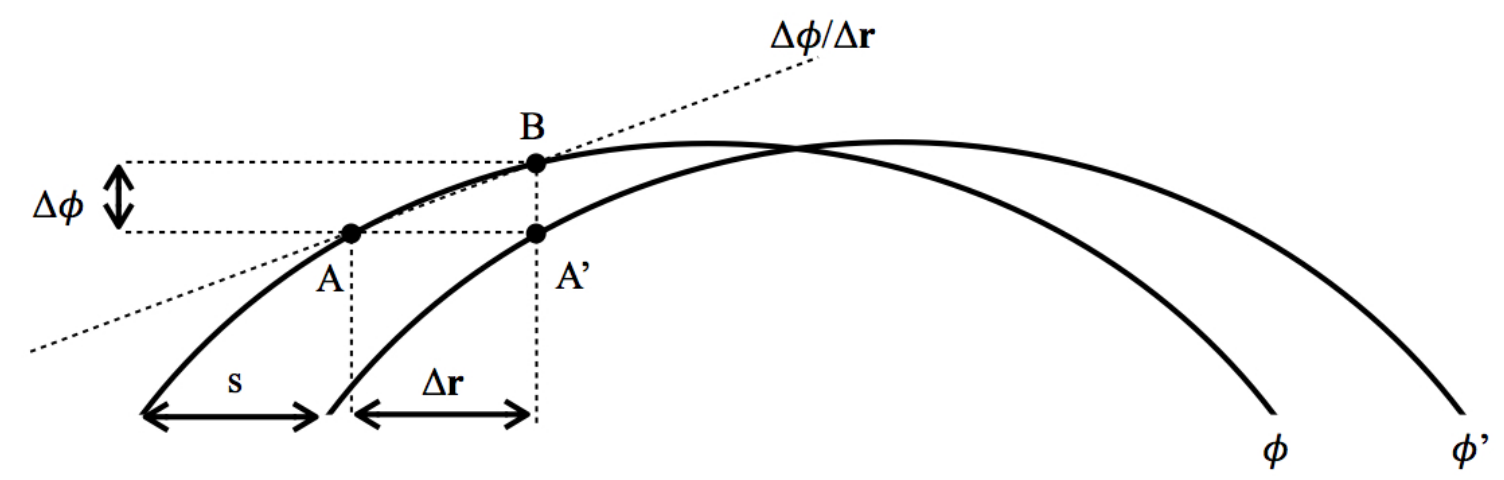

Figure 3.1: Wavefront $\phi$ interfering with a sheared version of itself, $\phi^{\prime}$.

In order to demonstrate why this method of interferometry provides information about the gradient of the wavefront we must look at the difference in phase $\Delta \phi$, between the unsheared $\phi(\vec{r})$ and sheared $\phi^{\prime}(\vec{r})$ wavefronts in one dimension and subsequently generalize to three dimensions. With one wavefront 
sheared in an arbitrary direction by the amount $s$, the wavefront difference is

$$
\begin{aligned}
\Delta \phi & =\phi(\vec{r})-\phi^{\prime}(\vec{r}) \\
& =\phi(\vec{r})-\phi(\vec{r}-\vec{s}) .
\end{aligned}
$$

Multiplying $\Delta \phi$ by $\Delta r / \Delta r$ and recognizing that the displacement of the two wavefronts is nothing more than the amount of shear between the two wavefronts $s=\Delta r$ allows us to write

$$
\Delta \phi \frac{\Delta r}{\Delta r}=s\left(\frac{\Delta \phi}{\Delta r}\right) .
$$

In the limit that the shear is small, we can express the term in parenthesis as the gradient of the wavefront in the direction of the shear

$$
\lim _{r \rightarrow 0} \frac{\Delta \phi}{\Delta r}=\frac{\partial \phi}{\partial r}=\nabla_{r} \phi
$$

Generalizing to three dimensions, we can write the resulting interference as the dot product between the gradient of the wavefront and the shear

$$
\Delta \phi=\nabla \phi \cdot \vec{s}
$$

In order to determine the intensity measured by a photodetector, we must determine the irradience at a point by squaring the sum of the electric fields arriving at this location. In this analysis, we use phasor notation and express the electric field in complex form $\widetilde{E}=E e^{i(\vec{k} \cdot \vec{r}-\omega t+\phi)}$. The intensity due to both electric fields $\left(\widetilde{E}_{t}=\widetilde{E}+\widetilde{E}^{\prime}\right)$ is

$$
\begin{aligned}
I_{S}(\vec{r}) & =\left(c n \epsilon_{0} / 2\right)\left|\widetilde{E}_{\text {total }}\right|^{2}=\left(c n \epsilon_{0} / 2\right)\left(\widetilde{E}_{t} \cdot \widetilde{E}_{t}^{*}\right)=\left(c n \epsilon_{0} / 2\right)\left[\left(\widetilde{E}+\widetilde{E}^{\prime}\right) \cdot\left(\widetilde{E}^{*}+\widetilde{E}^{\prime *}\right)\right] \\
& =\left(c n \epsilon_{0} / 2\right)\left[\left(\widetilde{E} \cdot \widetilde{E}^{*}\right)+\left(\widetilde{E}^{\prime} \cdot \widetilde{E}^{\prime *}\right)+\left(\widetilde{E} \cdot \widetilde{E}^{\prime *}\right)+\left(\widetilde{E}^{*} \cdot \widetilde{E}^{\prime}\right)\right] \\
& =I+I^{\prime}+\sqrt{I^{\prime} I}\left[e^{i\left(\vec{k} \cdot \vec{r}+\phi-\overrightarrow{k^{\prime}} \cdot \vec{r}-\phi^{\prime}\right)}+e^{-i\left(\overrightarrow{k_{1}} \cdot \vec{r}+\phi-\overrightarrow{k^{\prime}} \cdot \vec{r}-\phi_{2}\right)}\right] \\
& =I+I^{\prime}+2 \sqrt{I^{\prime} I} \cos \left(\vec{k} \cdot \vec{r}+\phi-\overrightarrow{k^{\prime}} \cdot \vec{r}-\phi^{\prime}\right)
\end{aligned}
$$


where $\vec{k}$ is the wave vector, $\vec{r}$ is the position vector, $\omega$ is the angular frequency of the light, and $I$ and $I^{\prime}$ are the intensity of the unsheared and sheared fields respectively. We see that the total intensity is related to the sum of the intensities of each incident electric field, as well as an interference term due to the mixing of the two fields.

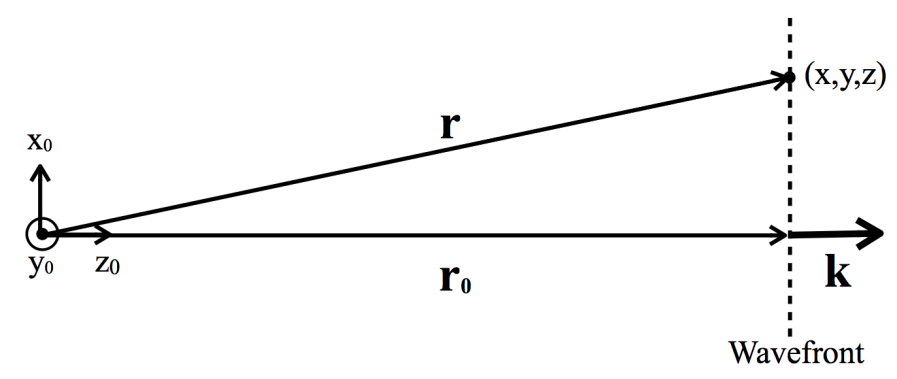

Figure 3.2: Coordinates used in the paraxial approximation.

If we consider a nearly flat wavefront as shown in Figure 3.2 , we can take advantage of some of the simplifying features of the paraxial approximation. This approximation allows us to consider the optical waves to be traveling in the $\hat{z}$ direction (i.e. $\vec{k}=\vec{k}^{\prime}=k \hat{z}$ ), and any transverse variation in phase can be included in $\phi$. Let us consider for a moment, only the argument of the interference term in equation 3.6, taking advantage of the paraxial approximation. That is to say $\vec{k} \rightarrow k \hat{z}$ and $\phi \rightarrow \phi(\vec{r})$,

$$
\begin{aligned}
\Delta \phi(\vec{r}) & =(\vec{k} \cdot \vec{r})-\left(\overrightarrow{k^{\prime}} \cdot \vec{r}\right)+\phi(\vec{r})-\phi^{\prime}(\vec{r}) \\
& =\phi(\vec{r})-\phi^{\prime}(\vec{r}) .
\end{aligned}
$$

Thus, the argument of the interference term contains only the difference between the transverse variations in phase. In shearing interferometry, the two phase fronts have the same spatial profile, and the interference is due to the displacement of one 
wavefront by an amount $s$

$$
\phi(\vec{r})-\phi^{\prime}(\vec{r})=\phi(\vec{r})-\phi(\vec{r}-\vec{s})
$$

which has exactly the same form as equation 3.2. Thus, the measured intensity is a function of the amount of shear and the gradient of the wavefront

$$
I_{s}(\vec{r})=I+I^{\prime}+2 \sqrt{I^{\prime} I} \cos (\nabla \phi \cdot \vec{s})
$$

\subsection{Shear Through Birefringence}

The previous analysis made no mention of the method used to shear the two wavefronts, and in fact, a variety of methods exist to do so. The method chosen for this design involves the use of a birefringent crystal to laterally shear two orthogonal polarizations relative to one another.

Maxwell's equations in electrically neutral, nonmagnetic solids can be expressed as

$$
\begin{aligned}
& \nabla \times \vec{E}=-\mu_{0} \frac{\partial \vec{H}}{\partial t} \\
& \nabla \times \vec{H}=\epsilon_{0} \frac{\partial \vec{E}}{\partial t}+\frac{\partial \vec{P}}{\partial t}+\vec{J} \\
& \nabla \cdot \vec{E}=-\frac{1}{\epsilon_{0}}(\nabla \cdot \vec{P}) \\
& \nabla \cdot \vec{H}=0
\end{aligned}
$$

where $\vec{E}$ is the electric field, $\vec{H}$ denotes the magnetic field, the polarizability is

denoted by $\vec{P}, \vec{M}$ is the magnetization and $\vec{J}$ is the current density. The wave equation for the electric field is then obtained in the usual way by taking the curl of equation 3.11

$$
\nabla \times(\nabla \times \vec{E})+\frac{1}{c^{2}} \frac{\partial^{2} \vec{E}}{\partial t^{2}}=-\mu_{0} \frac{\partial^{2} \vec{P}}{\partial t^{2}}-\mu_{0} \frac{\partial \vec{J}}{\partial t}
$$


The sources of the wave equation are contained in the terms on right hand side of Equation 3.15. In the case of a non-metal, the source of the wave equation is the polarizability, since the current density of such materials is negligible [9].

An optical wave traveling through a material can be modeled using the classical electron oscillator model, where the electric field of the incident optical wave drives the bound electrons in the material which then act as sources for the waves that then propagate through the material. The periodic structure of an anisotropic material (like some crystals) gives rise to unusual optical properties such as "birefringence," which is polarization-based phase shift. The electrons in a crystal are trapped in periodic potential wells formed by the regularly spaced atomic nuclei in the crystal. An electron trapped in an asymmetric potential well of a crystal lattice and excited by an incident electric field due to an optical wave can be modeled as a mass attached to several springs of differing spring constants $(K)$, as shown in Figure 3.3. When pushed in an arbitrary direction, the displacement of the mass will depend on the direction of the force relative to the principle axes of the system and the stiffness of the springs along each principle axis [9].

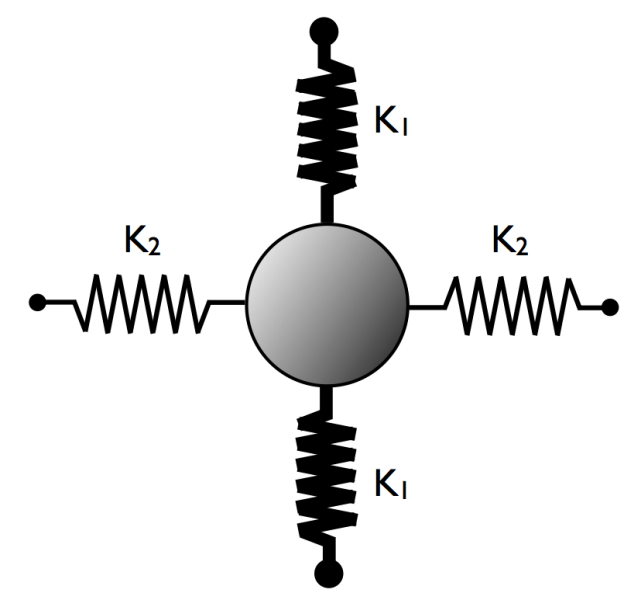

Figure 3.3: A mass attached to four springs with differing spring constants $(K)$ serves as a model for an electron in an asymmetric potential well of a crystal lattice. 
Returning to our physical system of electrons in a crystal lattice, the displacement of an electron depends on both the magnitude as well as the polarization of the driving electric field. We can define principle axes such that polarization of light along either of these axes remains unchanged as it passes through the crystal. Each principle axis has associated with it a particular phase velocity for an optical wave polarized along that axis. Thus, each polarization component will travel different optical path lengths within the crystal, resulting in phenomena such as "spatial walk-off" and "double refraction," where the two polarization components are refracted at different angles, and thus displaced from each other after traveling the length of the crystal $(L)$. Double refraction occurs for a non-normally incident optical wave, whereas spatial walk-off occurs for a normally incident wave, as shown in Figure 3.4. The walk off angle $\rho$ is the angle of deviation between the polarization components along the crystallographic principle axes denoted by $x^{\prime}, y^{\prime}$ and $z^{\prime}$ in Figure 3.4.



Figure 3.4: Spatial walk off (or double refraction) of a laser beam transmitted through a birefringent crystal of Beta Barium Borate (BBO). 
Our goal is to determine the amount of shear $s$ caused by spatial walk-off from traversing the birefringent crystal. We begin by expressing the electric displacement $\vec{D}$ in terms of the incident electric field $\vec{E}$ and the polarizibility of the material $\vec{P}$

$$
\vec{D}=\epsilon_{0} \vec{E}+\vec{P}
$$

The polarization of the material $\vec{P}$ depends on the incident electric field $\vec{E}$ as well as the electric susceptibility tensor $\bar{\chi}$. Expressing this in terms of the principle axes causes off-diagonal terms of the susceptibility tensor to vanish

$$
\begin{gathered}
\vec{P}=\epsilon_{0} \bar{\chi} \vec{E} \\
{\left[\begin{array}{c}
P_{x} \\
P_{y} \\
P_{z}
\end{array}\right]=\epsilon_{0}\left[\begin{array}{ccc}
\chi_{11} & 0 & 0 \\
0 & \chi_{22} & 0 \\
0 & 0 & \chi_{33}
\end{array}\right]\left[\begin{array}{c}
E_{x} \\
E_{y} \\
E_{z}
\end{array}\right] .}
\end{gathered}
$$

Combining Equations 3.16 and 3.17 provides a relationship between the electric displacement and the polarization of the incident electric field

$$
\vec{D}=\epsilon_{0}(1+\bar{\chi}) \vec{E}=\vec{\epsilon} \vec{E}
$$

The diagonal elements of the susceptibility tensor in equation 3.18 give rise to the index of refraction for an electric field polarized along a principle axis of the crystal $n_{i}=\sqrt{1+\chi_{i i}}$. A material whose susceptibility tensor has $\chi_{11}=\chi_{22} \neq \chi_{33}$ is referred to as a uniaxial birefringent crystal. Crystals of this type have an ordinary index of refraction given by $n_{o}=\sqrt{1+\chi_{11}}=\sqrt{1+\chi_{22}}$ and an extraordinary index of refraction $n_{e}=\sqrt{1+\chi_{33}}$. If $n_{o}>n_{e}$ the crystal is referred to as negative uniaxial whereas if $n_{e}>n_{o}$ it is a positive uniaxial crystal [9].

In this experiment, Beta Barium Borate $\left(\mathrm{BaB}_{2} \mathrm{O}_{4}\right)$, a negative uniaxial crystal, was used. The differing indices of refraction give rise to spatial walk off, where the 
two principle polarzations are deviated by different amounts when passing into the crystal.

In order to determine the amount of shear generated by passing through the crystal, we must determine the walk off angle $\rho$ between the two eigenpolarizations of the material. The walk off angle is given by angle between the electric field vector $\vec{E}$ and the electric displacement vector $\vec{D}[9]$

$$
\begin{gathered}
\vec{E} \cdot \vec{D}=|\vec{E}||\vec{D}| \cos (\rho) \\
{\left[\begin{array}{c}
D_{x} \\
D_{y} \\
D_{z}
\end{array}\right]=\epsilon_{0}\left[\begin{array}{ccc}
n_{o}^{2} & 0 & 0 \\
0 & n_{o}^{2} & 0 \\
0 & 0 & n_{e}^{2}
\end{array}\right]\left[\begin{array}{c}
E_{x} \\
E_{y} \\
E_{z}
\end{array}\right] .}
\end{gathered}
$$

The crystallographic axes for the particular cut of BBO used in this experiment are such that the y-axis is an eigenpolarization, so $D_{y}=\epsilon_{0} n_{o}^{2} E_{y}$, and the crystal's z-axis is rotated by $\theta$ from the surface normal. Equation 3.21 simplifies to a two-by-two matrix equation for the polarization component in the $x-z$ plane

$$
D\left[\begin{array}{c}
\cos (\theta) \\
\sin (\theta)
\end{array}\right]=\epsilon_{0}\left[\begin{array}{cc}
n_{o}^{2} & 0 \\
0 & n_{e}^{2}
\end{array}\right]\left[\begin{array}{c}
E_{x} \\
E_{z}
\end{array}\right],
$$

where

$$
\begin{aligned}
\vec{E} \cdot \vec{D} & =\frac{D^{2}}{\epsilon_{0}}\left[\frac{\cos ^{2} \theta}{n_{o}^{2}}+\frac{\sin ^{2} \theta}{n_{e}^{2}}\right] \\
D & =|\vec{D}| \\
|\vec{E}| & =\frac{D}{\epsilon_{0}}\left[\frac{\cos ^{2} \theta}{n_{o}^{4}}+\frac{\sin ^{2} \theta}{n_{e}^{4}}\right]^{1 / 2} .
\end{aligned}
$$

Thus, from equation 3.20, the walk off angle is

$$
\begin{aligned}
\rho & =\cos ^{-1}\left(\frac{\vec{E} \cdot \vec{D}}{|\vec{E}||\vec{D}|}\right) \\
& =\cos ^{-1}\left(\frac{\left[\frac{\cos ^{2} \theta}{n_{o}^{2}}+\frac{\sin ^{2} \theta}{n_{e}^{2}}\right]}{\sqrt{\frac{\cos ^{2} \theta}{n_{o}^{4}}+\frac{\sin ^{2} \theta}{n_{e}^{4}}}}\right) .
\end{aligned}
$$


After traveling a distance of L through the crystal, the two wavefronts are sheared an amount $s=L \tan (\rho)$, as shown in Figure 3.4.

Because the two polarizations states travel different optical path lengths through the BBO, we must account for a static phase shift $\Gamma$ between two corresponding points on the sheared and unsheared wavefronts in the interference term of Equation 3.10

$$
I_{s}(\vec{r})=I+I^{\prime}+2 \sqrt{I^{\prime} I} \cos (\nabla \phi \cdot \vec{s}+\Gamma) .
$$

The phase shift due to the static birefringence of the optics is common to the thermally deformed and undeformed wavefronts so it need not be explicitly known since it cancels out in the analysis.

\subsection{System Design}

In the design presented here, and shown schematically in Figure 3.5, a Helium-Neon laser polarized at $45^{\circ}$ acts as a probe to interrogate the surface of a gold coated one-inch diameter test optic whose thermal deformation can be controlled via a secondary violet pump laser. The gold coating strongly absorbs energy from the violet laser, while the BK7 glass substrate, chosen for its relatively low thermal conductivity and high thermal expansion coefficient, allows slow heat dissipation. This combination results in a relatively large local surface deformation on the front face of the test optic.

A microscope objective and pinhole clean the spatial mode of the laser before it reflects off the surface of the gold coated test optic. The light then passes through the BBO crystal which shears the wavefront. Since the relative amplitudes of the sheared wavefronts is polarization dependent, the probe beam must be polarized at $45^{\circ}$ in order to contain equal amplitudes along the principle axes of the BBO. A half 
wave $(\lambda / 2)$ plate placed after the crystal rotates the polarization once again so that each principle polarization state of the crystal has a component along the transmission axis of a fixed orientation polarizing beam splitter (labeled "PBS" in Figure 3.5) placed in front of the imaging sensor.

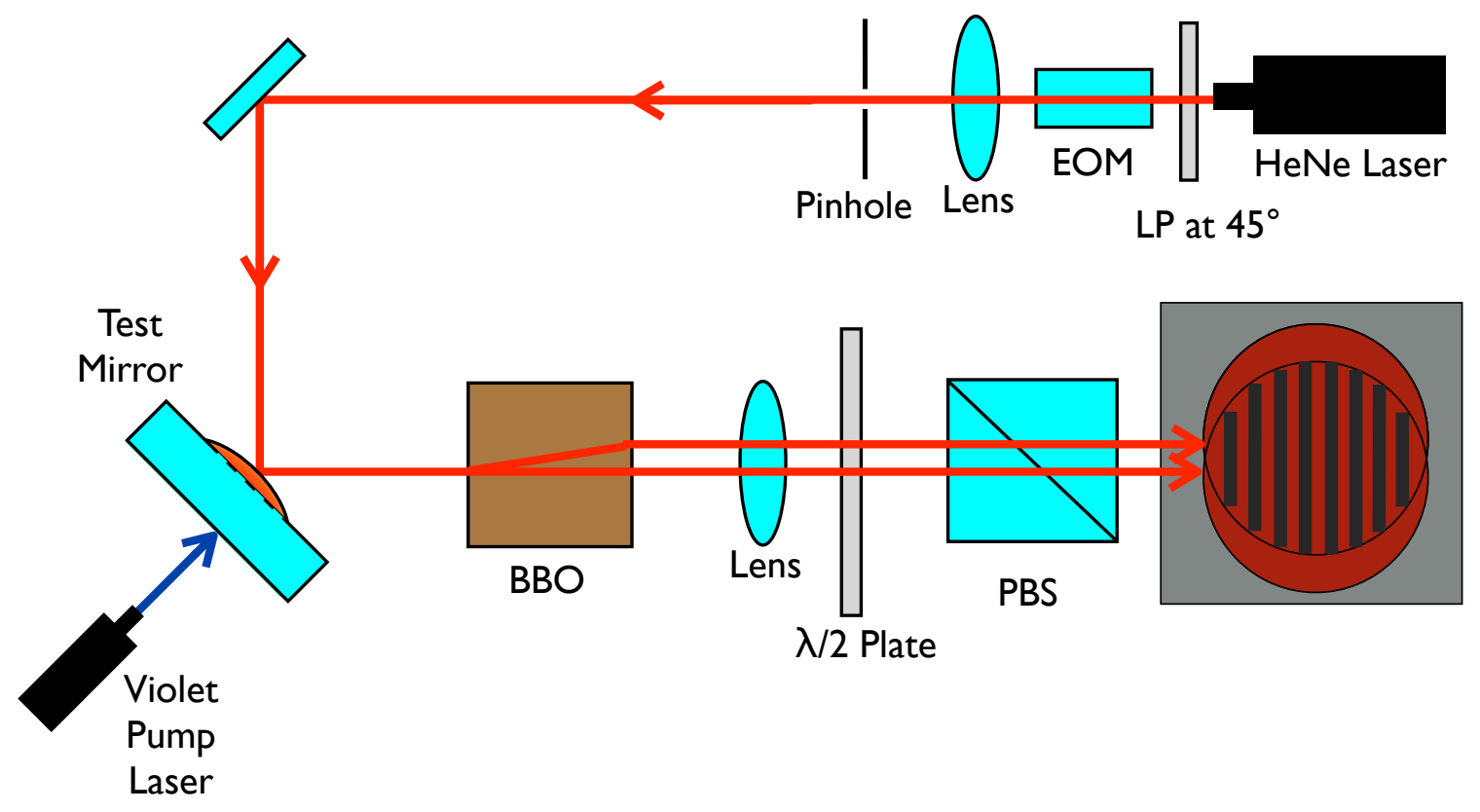

Figure 3.5: Schematic diagram of the shearing interferometer.

An electro-optic phase modulator consisting of a crystal of z-cut Lithium Niobate placed before the test optic (cosinusoidally) modulates the interference pattern, allowing the interference pattern to be isolated from the background light while a video of the time varying interference pattern is recorded.

In order to reconstruct the curvature of the wavefront in two dimensions, the interference pattern is first recorded with one orientation of the BBO crystal. The crystal orientation is then rotated by 90 degrees around the propagation axis of the beam to record the interferogram for the other component of the wavefront gradient. For each orientation, two videos were recorded, one recording the 
undistorted modulated wavefront, and the other recording the distorted modulated wavefront, requiring a total of four video recordings. Each video recording is processed to extract the component of the gradient in the direction of the shear.

\subsection{Modulation}

The interference pattern that results from the shearing interferometer is superimposed on any background light that illuminates the imaging sensor. Modulation of the interference pattern allows homodyne detection to determine the phase shift even in the presence of background light and without knowledge of the detector's response function. This is accomplished by using an electrooptic modulator placed at the output of the laser, oriented such that the ordinary and extraordinary waves have equal amplitudes. The birefringence of the electro-optic modulator can be controlled via an applied sinusoidal voltage to produce a sinusoidally varying advance or lag in the phase of one polarization component relative to the orthogonal polarization component. This time varying advance or lag between polarization components results in modulation of the interference pattern.

The electrooptic crystal used here, z-cut Lithium Niobate, has a voltage induced birefringence that depends on the crystal orientation and applied (cosinusoidal) voltage $\mathrm{V}$ such that

$$
\Gamma=\frac{2 \pi}{\lambda}\left(n_{e}-n_{0}\right) L-\frac{\pi}{\lambda}\left(n_{e}^{3} r_{33}-n_{o}^{3} r_{13}\right) \frac{L}{d} V
$$

where $L$ and $d$ are the length of the crystal and electrode separation respectively, $n_{o}$ and $n_{e}$ are the ordinary and extraordinary indices of refraction, and $r_{13}$ and $r_{33}$ are elements of the electrooptic tensor for Lithium Niobate [33].

The birefringence is modulated cosinusoidally so that $\Gamma=\Gamma_{0}+m \cos (\omega t)$, where $\Gamma_{0}$ represents any static birefringence in the experiment. The relative 
magnitude of the interference term in equation 3.26 is proportional to

$$
\cos \left(\nabla \phi \cdot \vec{s}+\Gamma_{0}+m \cos (\omega t)\right)=\cos (\psi+m \cos (\omega t))
$$

where we have adopted the notation $\psi=\nabla \phi \cdot \vec{s}+\Gamma_{0}$ to define explicitly the time independent parameters. This can be expressed using the sum angle formula for cosines as:

$$
\begin{aligned}
\cos (\psi+m \cos (\omega t)) & =\cos (\psi) \cos (m \cos (\omega t)) \\
& -\sin (\psi) \sin (m \cos (\omega t)) .
\end{aligned}
$$

Equation 3.29 can be further expanded as a series of sinusoidal functions each with Bessel function amplitudes by using the Jacobi-Anger expansion:

$$
\begin{aligned}
\cos (\psi+m \cos (\omega t)) & =\cos (\psi)\left[J_{0}(m)+2 \sum_{n=1}^{\infty}(-1)^{n} J_{2 n}(m) \cos (2 n \omega t)\right] \\
& -\sin (\psi)\left[-2 \sum_{n=1}^{\infty}(-1)^{n} J_{2 n-1}(m) \cos ((2 n-1) \omega t)\right] .
\end{aligned}
$$

If we determine the modulation depth $m$ and the amplitude $A$ of an even and odd frequency harmonic, we can solve for the phase shift $\psi$, which depends on the gradient of the wavefront. Writing only the first and second order harmonics corresponding to $n=1$

$$
\begin{aligned}
\cos (\psi+m \cos (\omega t)) & =\cos (\psi) J_{0}(m)-\left[2 \cos (\psi) J_{2}(m)\right] \cos (2 \omega t) \\
& -\left[2 \sin (\psi) J_{1}(m)\right] \cos (\omega t)+\ldots \\
& =\cos (\psi) J_{0}(m)-A_{2} \cos (2 \omega t)-A_{1} \cos (\omega t)+\ldots
\end{aligned}
$$


We define the amplitude of the first three harmonic frequency components as

$$
\begin{aligned}
& A_{1}=-2 \sin (\psi) J_{1}(m) \\
& A_{2}=-2 \cos (\psi) J_{2}(m) \\
& A_{3}=2 \sin (\psi) J_{3}(m) .
\end{aligned}
$$

The modulation period can be determined numerically by computing the frequency of the lowest non-zero peak in the temporal fast Fourier transform for each pixel over 30 seconds of video data. The modulation depth $m$ can be determined numerically from the ratio of the amplitude of the fundamental $A_{1}$ and third harmonic $A_{3}$

$$
\frac{A_{1}}{A_{3}}=-\frac{J_{1}(m)}{J_{3}(m)} .
$$

$A_{1} / A_{3}$ is calculated pixel-by-pixel and subsequently averaged. The modulation depth is then the best fit value of $m$ for which $J_{1}(m) / J_{3}(m)$ most closely matches the average value of $A_{1} / A_{3}$.

\subsection{Wavefront Reconstruction}

Shearing interferometers are much easier to align and provide excellent common mode noise rejection compared to most other types of interferometers, but this comes at the cost of a more complicated interferogram processing scheme. Since the detected interferogram contains information about the gradient of the wavefront, rather than information about displacement, a more complex analysis is required compared to typical interferometric schemes.

Taking the ratio of the coefficients of the first and second order harmonic 
terms, $A_{1}$ and $A_{2}$ in Equation 3.30, allows us to solve for the phase $\psi=\nabla \phi \cdot \vec{s}+\Gamma_{0}$

$$
\begin{aligned}
\frac{A_{1}}{A_{2}} & =\frac{(\sin \psi) J_{1}(m)}{(\cos \psi) J_{2}(m)} \\
\psi & =\tan ^{-1}\left(\frac{J_{2}(m) A_{1}}{J_{1}(m) A_{2}}\right)
\end{aligned}
$$

where $A_{1}$ is the magnitude of the temporal Fourier transform at angular frequency $\omega$ and $A_{2}$ is the magnitude at $2 \omega$. The measured phase can then be related to the wavefront gradient $\nabla \phi$ and the shear $\vec{s}$ according to

$$
\nabla \phi \cdot \vec{s}=\tan ^{-1}\left(\frac{J_{2}(m) A_{1}}{J_{1}(m) A_{2}}\right)-\Gamma_{0}
$$

where $\Gamma_{0}$ is a constant term that represents any phase shift due to birefringence of the optics and is irrelevant for our analysis since it cancels out when taking the difference between the deformed and undeformed wavefronts.

One problem remains because $\psi$, which is calculated from a four quadrant inverse tangent function (equation 3.37), is cyclic in $2 \pi$. The resulting ambiguity in phase is reconciled using a quality guided 2D "unwrapping" algorithm which ensures there are no discontinuities in phase between adjacent pixels which are greater than $\pi$ in the reconstructed wavefront [10].

To minimize errors caused by the unwrapping process, we first find the phase difference between the distorted and reference wavefronts, denoted by subscript $d$ and subscript $r$ respectively, and only unwrap the difference making use of the difference formula for arctangents [2]

$$
\begin{aligned}
\psi_{d}-\psi_{r} & =\tan ^{-1}\left(z_{d}\right)-\tan ^{-1}\left(z_{r}\right) \\
& =\tan ^{-1}\left(\frac{z_{d}-z_{r}}{1+z_{d} z_{r}}\right) .
\end{aligned}
$$


This is the deformation induced change in the gradient of the wavefront

$$
\begin{aligned}
\psi_{d}-\psi_{r} & =\left(\Gamma_{0}+\nabla \phi_{d} \cdot \vec{s}\right)-\left(\Gamma_{0}+\nabla \phi_{r} \cdot \vec{s}\right) \\
& =\nabla\left(\phi_{d}-\phi_{r}\right) \cdot \vec{s} \\
& =\nabla \delta \cdot \vec{s}
\end{aligned}
$$

where $\delta \equiv \phi_{d}-\phi_{r}$ is the difference in phase induced by the surface deformation.

Once the wavefront deformation gradient in both directions $(x$ and $y)$ is determined, the components of the surface deformation gradient can be determined from the measured wavefront deformation gradient:

$$
2 k \nabla h=\nabla \delta
$$

where the factor of two arises from the round trip path length difference in accumulated phase and $k=2 \pi / \lambda$. The mean value of $\nabla h$ is subtracted in order to remove any tilt of the wavefront, since it is irrelevant for our analysis and the partial derivatives, $\partial h(\vec{r}) / \partial x$ and $\partial h(\vec{r}) / \partial y$ are shifted by the average lateral displacement, $-s / 2$. The surface deformation is assumed to be localized, providing information about the deformation at the reflecting surface of the mirror. This assumption allows us to use a Poission reconstruction with Neumann boundary conditions to reconstruct the mirror's surface profile $h(\vec{r})$.

Reconstruction of surfaces from measured gradient fields has become an important topic in other areas of research, and reliable algorithms are readily available. We employed an algebraic surface reconstruction algorithm described in [3] to reconstruct the mirror surface measured here. 


\subsection{Sensitivity and Resolution}

The sensitivity of the shearing interferometer is estimated from the spatial noise spectrum of the processed interferogram and is determined from the interferogram recorded for shear in one direction. The 1D Fourier transform of the central row of pixels is computed and used as a representative noise spectrum. We can separate the contribution from the optical magnification and the shear on this noise level by defining

$$
d k^{\prime}=2 \pi s / x,
$$

with $s$ the magnitude of the shear and $x$ the width of the region of the surface being measured. With the spatial scaling effects confined to the term $d k^{\prime}$, we can express the sensitivity to a gradient in dimensionless units allowing this result to be scaled to account for the change in sensitivity with different optical magnification and/or shear. This can be compared to the peak of the gradient calculated from the analytical model.

Since the gradient scales inversely with the pump beam radius $\omega$ and the measured signal scales linearly with the amount of shear $s$, equivalent displacement sensitivities can be achieved for thermal deformations of differing spatial scale as long as the size of the shear is also scaled proportionally, limited only by $x$ the field of view of the image.

The theoretical displacement sensitivity of this interferometer is limited by the quantization noise of the n-bit imaging sensor. With the birefringence set to $\Gamma_{0}=\pi / 2$, equivalent to biasing the interference to the side of a fringe where the sensitivity is maximized (and linear), the minimum detectable value for $A_{2}$ in equation 3.38 is $A_{1} / 2^{n}$. The modulation depth produces a value of $J_{2}(m) / J_{1}(m)$ 
close to unity, so equation 3.38 gives

$$
\begin{aligned}
\nabla \phi \cdot \vec{s} & =\tan ^{-1}\left(\frac{J_{2}(m) A_{1} 2^{n}}{J_{1}(m) A_{1}}\right)-\frac{\pi}{2} \\
& \approx \tan ^{-1}\left(2^{n}\right)-\pi / 2
\end{aligned}
$$

Let us consider for a moment $\tan (\pi / 2-\alpha)$, where $\alpha=1 / 2^{n}$. This can be expressed in terms of sin and cos as

$$
\tan (\pi / 2-\alpha)=\frac{\sin (\pi / 2-\alpha)}{\cos (\pi / 2-\alpha)}=\frac{\cos (\alpha)}{\sin (\alpha)}
$$

Since $\alpha$ is small, we can use the small angle approximation to write

$$
\begin{aligned}
\tan (\pi / 2-\alpha) & \approx \frac{1-\alpha^{2} / 2}{\alpha} \\
& \approx 1 / \alpha-\alpha / 2 \\
& \approx 1 / \alpha
\end{aligned}
$$

which can be expressed in terms of our original quantity $2^{n}$ as

$$
\begin{aligned}
& \tan \left(\pi / 2-1 / 2^{n}\right) \approx 2^{n} \\
& \tan ^{-1}\left(2^{n}\right)-\pi / 2 \approx 1 / 2^{n} .
\end{aligned}
$$

Thus, the minimum detectable gradient is related to the number of bits in our imaging sensor

$$
(\nabla \phi \cdot \vec{s})_{\min } \approx \frac{1}{2^{n}}
$$

Relating this to the mirror deformation over a distance $s$, using $2 k \Delta h=\nabla \phi \cdot \vec{s}$, where $\Delta h$ is the height of the deformation, gives a displacement sensitivity of

$$
\Delta h_{\min }=\frac{\lambda}{4 \pi 2^{n}} .
$$


The reconstruction algorithm used in this scheme assumes the gradient of the wavefront can be approximated by finite differences between points on the wavefront separated by the shear distance $s$. This limits the spatial resolution of the instrument to $s$. We can approximate the wavefront $\phi(\vec{r})$ by a second order Taylor series expansion

$$
\phi\left(\overrightarrow{r_{0}}+\vec{s}\right) \approx \phi\left(\overrightarrow{r_{0}}\right)+s \phi^{\prime}\left(\overrightarrow{r_{0}}\right)+\frac{s^{2}}{2} \phi^{\prime \prime}\left(\overrightarrow{r_{0}}\right)
$$

Where $r_{0}$ is the point of interest on one of the wavefronts. Applying the Taylor expansion above to the interference term in equation 3.8 gives

$$
\begin{aligned}
\Delta \phi & =\phi\left(\overrightarrow{r_{0}}\right)-\phi\left(\overrightarrow{r_{0}}+\vec{s}\right) \\
& \left.\approx \phi\left(\overrightarrow{r_{0}}\right)-\left[\phi\left(\overrightarrow{r_{0}}\right)+s \phi^{\prime}\left(\overrightarrow{r_{0}}\right)+\frac{s^{2}}{2} \phi^{\prime \prime}\left(\overrightarrow{r_{0}}\right)\right)\right] .
\end{aligned}
$$

The assumptions in the reconstruction algorithm are valid for small shear such that $s \ll \phi^{\prime} / \phi$, so

$$
\begin{aligned}
\frac{s^{2}}{2} \phi^{\prime \prime}\left(\overrightarrow{r_{0}}\right) & \ll s \phi^{\prime}\left(\overrightarrow{r_{0}}\right) \\
s & \ll \frac{2 \phi^{\prime}\left(\overrightarrow{r_{0}}\right)}{\phi^{\prime \prime}\left(\overrightarrow{r_{0}}\right)} .
\end{aligned}
$$

Where $\phi^{\prime}$ is the gradient and $\phi^{\prime \prime}$ is the curvature (both) in the direction of shear. In the regime of this approximation, equation 3.57 reduces to

$$
\Delta \phi \approx-s \phi^{\prime}\left(\overrightarrow{r_{0}}\right)
$$

and the magnitude of the measured interference term is linearly proportional to the shear $s$, so the sensitivity also improves linearly with $\mathrm{s}$.

We can define a characteristic length $l_{c}=2 \phi^{\prime} / \phi^{\prime \prime}$ such that the shear must be slightly smaller than $l_{c}$ in order to obtain the best sensitivity while still obtaining sufficient spatial resolution to measure the deformation. Prior knowledge about the 
shape and magnitude of the wavefront distortion are beneficial in order to determine the characteristic length and thus the optimal shear. Fortunately, thermo-elastic deformations in mirror substrates is well understood and has been investigated extensively, as demonstrated in Chapter 2.

Shearing interferometers have a non-uniform spatial frequency response that is well understood [23]. The sampling of the phase difference between two points on the wavefront is insensitive to spatial fluctuations with an integer multiple of cycles within the shear distance, so surface features smaller than the shear distance can not be resolved.

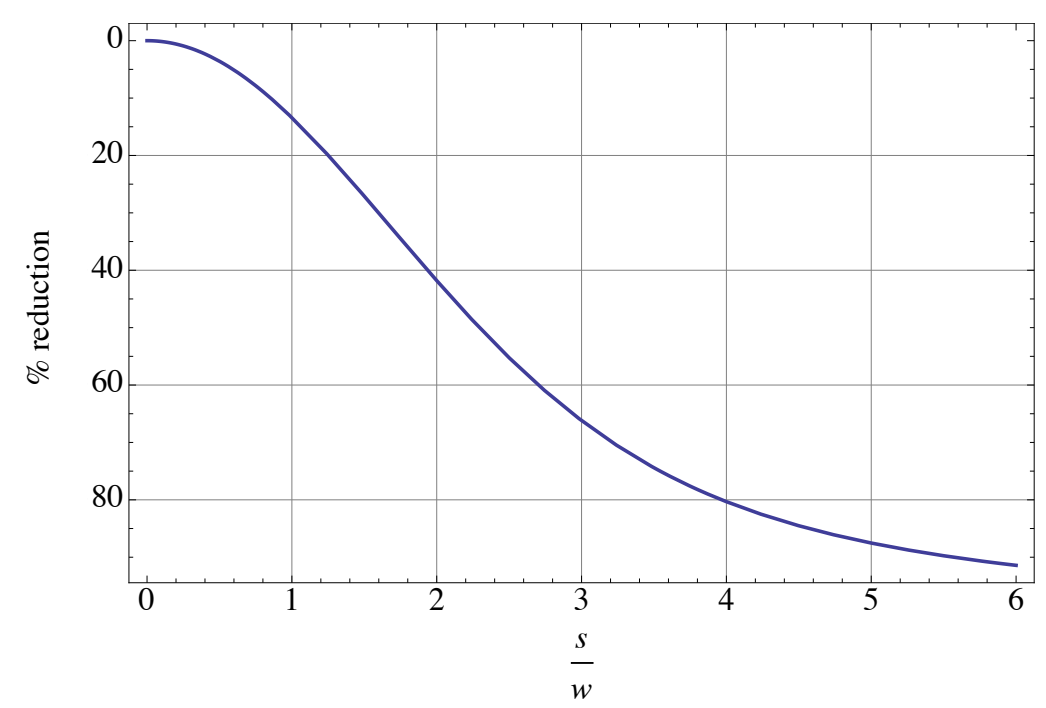

Figure 3.6: Percent reduction in the measured peak surface deformation due to spatial filtering from the shearing interferometer over a spatial length scale $s$.

The data analysis presented here does not attempt to account for this non-uniform spatial frequency response of the shearing interferometer because of the infinite values in the inverse filter necessary to fully compensate for the frequency response. Instead, we use the fact that the surface profile being investigated is 
known both through calculation and independent measurement using a Michelson interferometer, so the effect of the frequency response filter can be calculated and its effect on the measurement of the peak distortion can be used to compensate the measured value of the peak distortion.

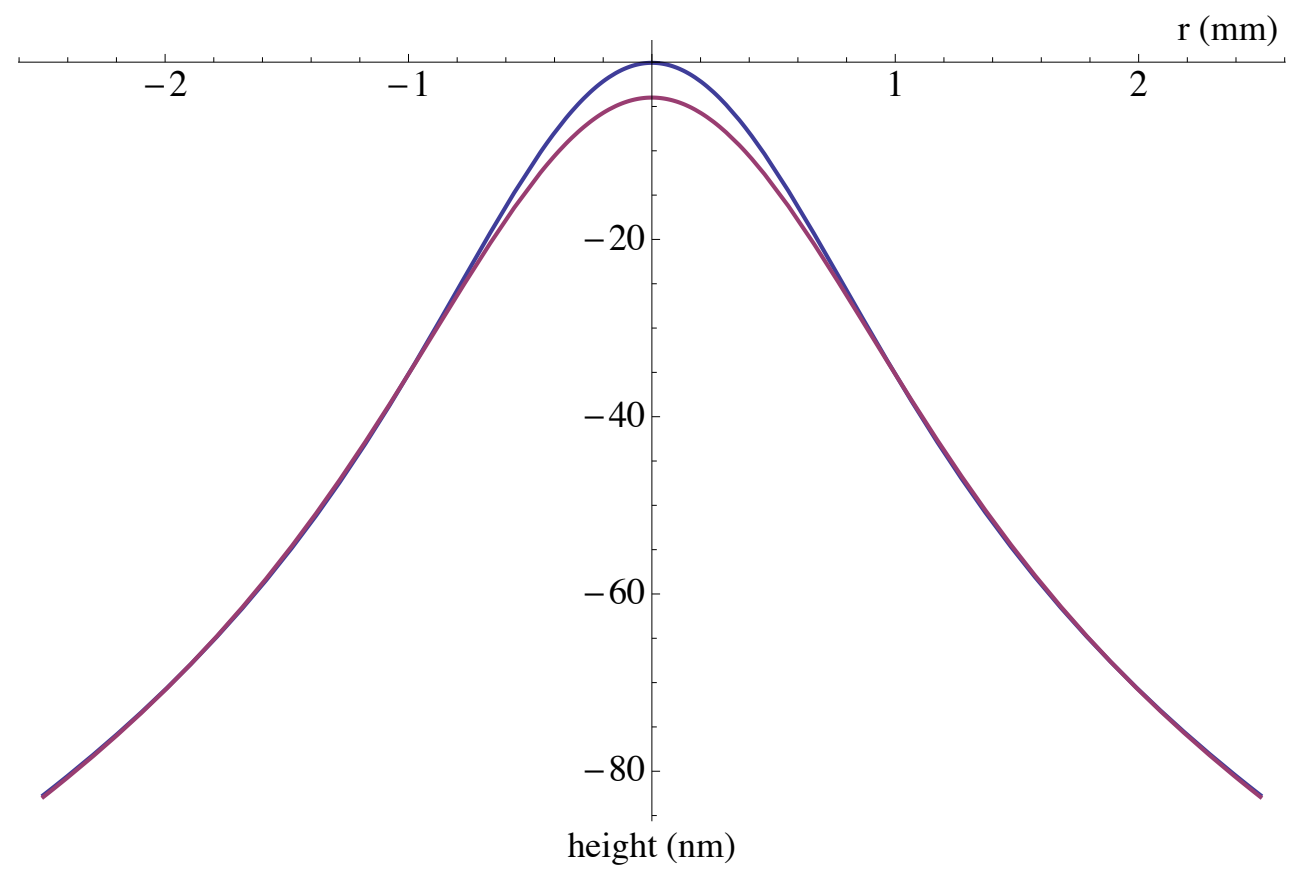

Figure 3.7: Theoretical bump height (blue) and the reduced height measured by the shearing interferometer (purple).

From the analytical expression for the surface gradient obtained in Chapter 2 (Equation 2.19) we can estimate the effect of the shearing interferometer's spatial frequency response on the overall magnitude of the reconstructed wavefront, which is shown in Figure 3.7. The fractional reduction in the magnitude of the thermal deformation due to spatial filtering from the interferometer was estimated as

$$
\frac{h_{\text {model }}(0)-h_{\text {measured }}(0)}{h_{\text {model }}(0)} \approx 1-\frac{1}{\phi(0)} \int_{-w}^{0} \frac{\phi(r+s / 2)-\phi(r-s / 2)}{s} d r
$$


where the surface deformation is evaluated over one Gaussian radius $w$ of the pump beam. This approximation is necessary because the analytical model used assumes an infinite mirror radius with an infinite surface displacement. Expression 3.60 is used to calculate the percent reduction in the measured peak surface deformation due to spatial filtering from the shearing interferometer over a spatial length scale $s$, shown in Figure 3.7. 


\section{CHAPTER 4}

\section{THERORETICAL BACKGROUND OF MICHELSON INTERFEROMETERY USED TO CONFIRM SHEARING MEASUREMENTS}

\subsection{Michelson Interferometer Design}

The off-axis design of the shearing interferometer lends itself well to simultaneous measurements using a Michelson interferometer, shown in Figure 4.1, as a means of confirming the results from the shearing interferometer. Since the interference pattern in a Michelson interferometer is sensitive to the differential path length between the two arms, it is well suited to measuring deformations in optics with minimal processing. The analysis of the Michelson interferograms described here has been adapted from Brooks [7].

The interference condition for a Michelson interferometer follows a similar analysis to that in section 3.1, Equation 3.6 yields the point by point intensity $I_{M}$

$$
\begin{aligned}
I_{M} & =\frac{c n \epsilon_{0}}{2}\left|\widetilde{E}_{\text {total }}\right|^{2} \\
& =I_{t}+I_{r}+\sqrt{I_{t} I_{r}}\left[e^{i\left(\phi_{t}-\phi_{r}\right)}+e^{-i\left(\phi_{t}-\phi_{r}\right)}\right] \\
& =I_{t}+I_{r}+2 \sqrt{I_{t} I_{r}} \cos (\Delta \phi),
\end{aligned}
$$

where $\phi_{r}$ is the phase acquired by light traveling in the reference arm of the interferometer, $\phi_{t}$ is the phase acquired by the light in the test arm, $\Delta \phi$ is the accumulated phase difference, and $I_{t}$ and $I_{r}$ are the intensity of the light from the test and reference arms respectively. The phase acquired by the light traveling one round trip in the reference arm is $\phi_{r}=2 k L_{r}$ where $L_{r}$ is the length from the 
beamsplitter to the mirror. The phase acquired by the light traveling one round trip in the test arm, which contains the thermally deformable mirror, is $\phi_{t}=2 k L_{t}-2 k \delta$, where $\delta$ denotes the height of the thermal deformation at a point. Thus, the accumulated phase difference is

$$
\begin{aligned}
\Delta \phi & =\phi_{t}-\phi_{r} \\
& =\left[2 k L_{t}-2 k \delta\right]-2 k L_{r} \\
& =k(2 \Delta L-2 \delta) .
\end{aligned}
$$



Figure 4.1: Schematic diagram of the Michelson interferometer used in this experiment. 
The arm length mismatch $\Delta L$ is common to both the deformed and undeformed interferograms so it is unnecessary that it be known explicitly for the analysis presented here.

In the presence of thermal deformation of the test optic, the differential arm length will vary slightly as a function of position on the surface by an amount $\delta$. The measured intensity $I_{M}$ will then be

$$
\begin{aligned}
I_{M} & =I_{1}+I_{2}+2 \sqrt{I_{1} I_{2}} \cos [k(2 \Delta L-2 \delta)] \\
& =I_{1}+I_{2}+\sqrt{I_{1} I_{2}}\left[e^{i k(2 \Delta L-2 \delta)}+e^{-i k(2 \Delta L-2 \delta)}\right]
\end{aligned}
$$

where $h$ represents the background intensity

$$
h=I_{1}+I_{2}
$$

and the interference term is denoted by

$$
\begin{aligned}
u+u^{*} & =\sqrt{I_{1} I_{2}} \exp [i k(2 \Delta L-2 \delta)] \\
& +\sqrt{I_{1} I_{2}} \exp [-i k(2 \Delta L-2 \delta)]
\end{aligned}
$$

We can generalize the above analysis to obtain $I_{M}(x, y)$, the measured intensity across the beam profile, to obtain

$$
I_{M}(x, y)=h(x, y)+u(x, y)+u^{*}(x, y)
$$

Information about the shape of the wavefront can be extracted from the spatial frequency spectrum of $I(x, y)$ by taking the Fourier transform, denoted by $\mathcal{F}\{\}$. In general, the Fourier transform of an arbitrary function $g(x, y)$, denoted by $G\left(K_{x}, K_{y}\right)$ is

$$
\begin{aligned}
G\left(K_{X}, K_{y}\right) & \equiv \mathcal{F}\{g(x, y)\} \\
& =\iint_{-\infty}^{+\infty} g(x, y) e^{-i\left(K_{x} x+K_{y} y\right)} d x d y
\end{aligned}
$$


where $K_{x}$ and $K_{y}$ are the spatial frequency components of the interference pattern $[26,20]$. The spectral components of equation 4.10 are thus

$$
\begin{aligned}
\mathcal{F}\left\{I_{M}(x, y)\right\} & =\mathcal{F}\left\{h+u+u^{*}\right\} \\
& =H\left(K_{x}, K_{y}\right)+U\left(K_{x}, K_{y}\right)+U^{*}\left(K_{x}, K_{y}\right) .
\end{aligned}
$$

However, equation 4.13 illustrates a problem that may not be readily apparent: all three of the Fourier transforms $H\left(K_{x}, K_{y}\right), U\left(K_{x}, K_{y}\right)$ and $U^{*}\left(K_{x}, K_{y}\right)$ have spatial frequencies centered around $K=0$. That is to say, the spatial frequency spectra of the background intensity distribution and the interference distribution (which contains the desired phase information about the wavefront) all overlap making the three terms inseparable; the signal can not be distinguished from the background.

\subsection{Spatial Frequency Modulation}

Modulating the interference signal with a spatial carrier frequency $K_{c}$ can serve to separate the center frequency of the phase information encoded in the interference terms from the background intensity which remains centered around $K=0$. The mirror tilt acts to phase modulate the interference pattern, causing dark and bright interference fringes which occur with spatial frequency $K_{c}$. An example of phase modulation is shown in Figure 4.2, where a gaussian shaped signal $u(x)$ (top graph, solid blue line), is used to phase modulate a cosinusoidal carrier function (bottom graph, dashed line) to give a phase modulated signal (bottom, solid blue line).

Modulation of the interference pattern can be achieved by slightly tilting one of the mirrors in the $x$ direction by an amount $\theta_{x}$, producing vertical interference fringes whose intensity vary cosinusoidally and whose spatial period is $X$. Constructive interference occurs when the (tilt-induced) additional path length is an 
integer multiple of the wavelength $2 X \theta_{x}=\lambda$. The spatial frequency of the interferogram is $K_{c}=2 \pi / X$ and can be expressed in terms of the wavelength and tilt of the mirror as $K_{c}=4 \pi \theta_{x} / \lambda$.
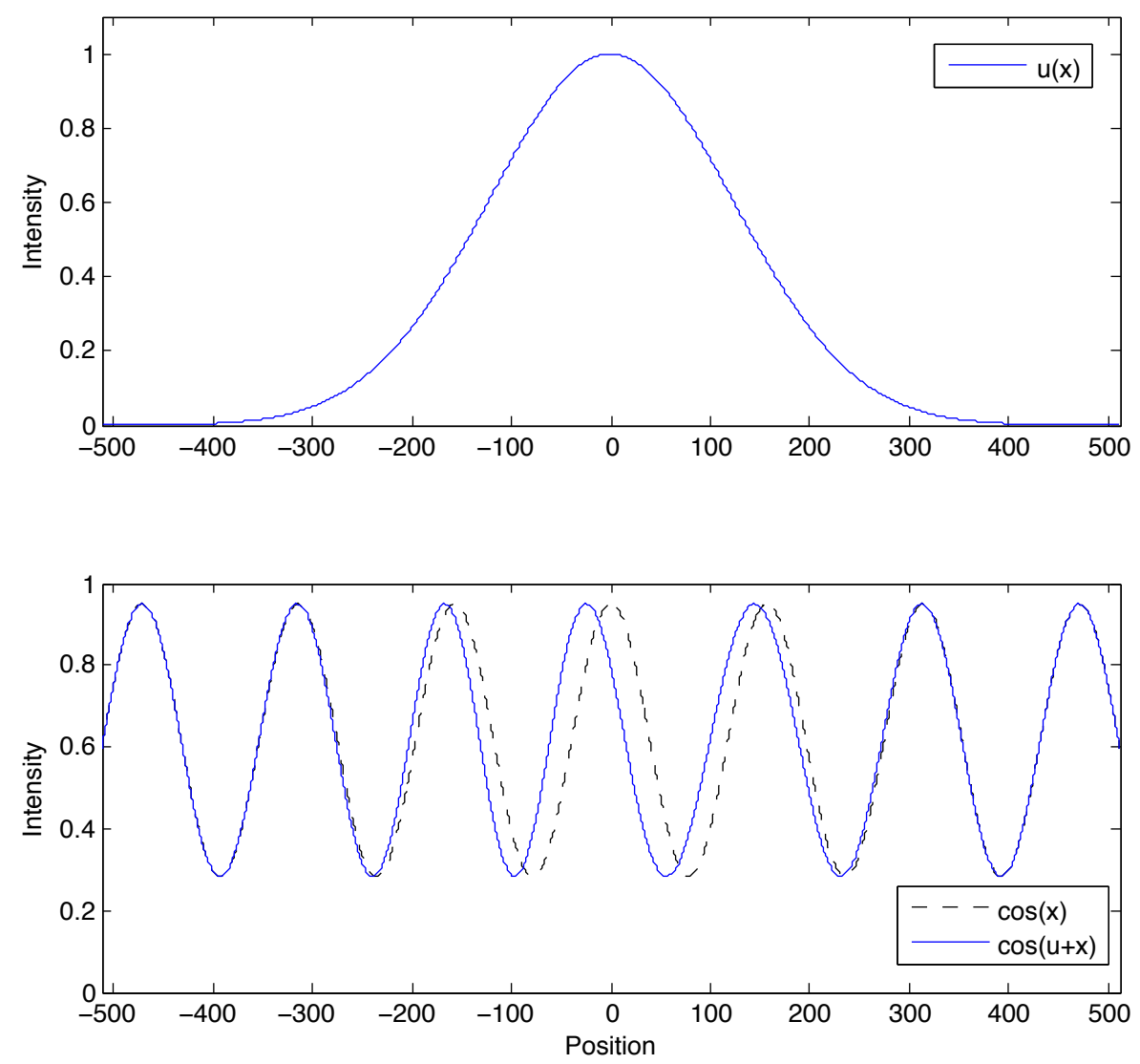

Figure 4.2: An example of phase modulation where a gaussian shaped signal is used to phase modulate a cosinusoidal carrier function.

The interference pattern $I^{\prime}(x, y)$ captured on the screen is thus

$$
\begin{aligned}
I_{M}^{\prime}(x, y) & =I_{1}+I_{2}+2 \sqrt{I_{1} I_{2}} \cos \left[k\left(2 \Delta L-2 \delta+2 \theta_{x} x\right)\right] \\
& =h(x, y)+u^{\prime}(x, y)+u^{\prime *}(x, y) .
\end{aligned}
$$

Now, the interference terms are multiplied by a term containing information about 
the spatial modulation

$$
\begin{aligned}
u^{\prime}(x, y) & =\sqrt{I_{1} I_{2}} \exp \left[i k\left(2 \Delta L-2 \delta+2 x \theta_{x}\right)\right] \\
& =u(x, y) e^{i k 2 x \theta_{x}}
\end{aligned}
$$

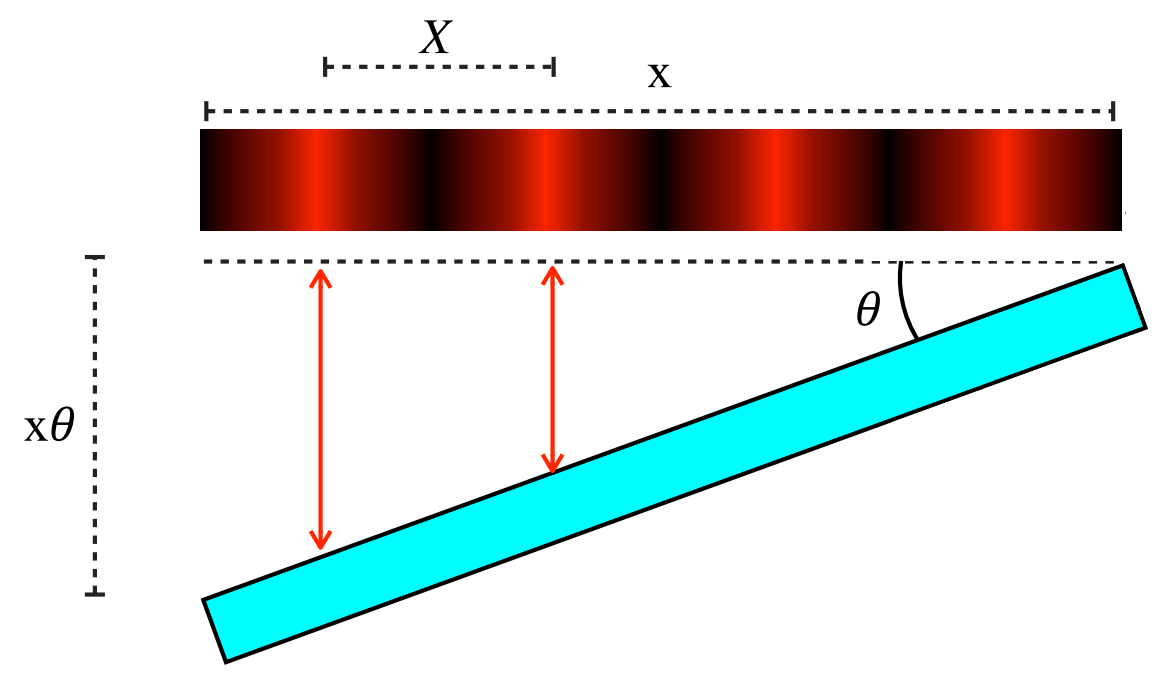

Figure 4.3: Schematic diagram of the tilted mirror in the Michelson interferometer which imposes a spatial carrier frequency on the measured intensity from the interferometer.

Taking the Fourier transform as before, we obtain

$$
\begin{aligned}
\mathcal{F}\left\{I_{M}^{\prime}(x, y)\right\} & =\mathcal{F}\left\{h+u^{\prime}+u^{\prime *}\right\} \\
& =\mathcal{F}\{h\}+\mathcal{F}\left\{u e^{i k 2 x \theta_{x}}\right\}+\mathcal{F}\left\{u^{*} e^{-i k 2 x \theta_{x}}\right\} \\
& =\mathcal{F}\{h\}+\mathcal{F}\{u v\}+\mathcal{F}\left\{u^{*} v^{*}\right\}
\end{aligned}
$$

Invoking the convolution theorem, which states that the Fourier transform of the product of two functions $u(x)$ and $v(x)$ equals the convolution of their Fourier transforms denoted by $U(K) \otimes V(K)$

$$
\mathcal{F}\{u(x) v(x)\}=U(K) \otimes V(K)
$$


where

$$
\begin{aligned}
V(K) & =\mathcal{F}\left\{e^{i k 2 x \theta_{x}}\right\} \\
& =\int_{-\infty}^{+\infty} e^{-i 2 \pi x\left(K-k 2 \theta_{x}\right)} d x \\
& =\int_{-\infty}^{+\infty} e^{-i 2 \pi x\left[K-\left(4 \pi \theta_{x} / \lambda\right)\right]} d x \\
& =\int_{-\infty}^{+\infty} e^{-i 2 \pi x\left(K-K_{c}\right)} d x=\delta\left(K-K_{c}\right) .
\end{aligned}
$$

Similarly,

$$
V^{*}(K)=\delta\left(K+K_{c}\right)
$$

and we see that the Fourier transform of the spatial modulation signal is the Dirac delta function, so the right hand side of equation 4.18 becomes

$$
\begin{aligned}
U(K) \otimes V(K) & =U(K) \otimes \delta\left(K-K_{c}\right) \\
& =U\left(K-K_{c}\right) .
\end{aligned}
$$

We now have all the pieces in place to obtain the frequency spectrum of the measured intensity of the spatially modulated (banded) interferogram

$$
\begin{aligned}
\mathcal{F}\left\{I_{M}^{\prime}(x, y)\right\} & =\mathcal{F}\{h\}+\mathcal{F}\{u v\}+\mathcal{F}\left\{u^{*} v^{*}\right\} \\
& =H(K)+U\left(K-K_{c}\right)+U^{*}\left(K+K_{c}\right),
\end{aligned}
$$

which all occur at different center frequencies in spatial frequency space, allowing us to isolate the desired low spatial frequency phase information contained in $U$ and $U^{*}$ at frequencies upshifted and downshifted by the modulation frequency $K_{c}$ from the low frequency background intensity centered around zero frequency as shown in the top graph of Figure 4.4 .

Since we are only interested in low spatial frequency modulation, further processing requires we filter out all components of the Fourier transform that do not 
occur near $K=K \pm K_{c}$ using a simple notch filter, as shown in the middle graph of Figure 4.4. The remaining frequency components are then shifted to zero frequency, completely equivalent to demodulation, as shown in the bottom graph of Figure 4.4, and the inverse Fourier transform (denoted by $\mathcal{F}^{-1}\{\}$ ) is calculated

$$
\begin{aligned}
\mathcal{F}^{-1}\left\{U(K)+U^{*}(K)\right\} & =u+u^{*} \\
& =e^{i k(2 \Delta L-2 \delta)}+e^{-i k(2 \Delta L-2 \delta)} .
\end{aligned}
$$
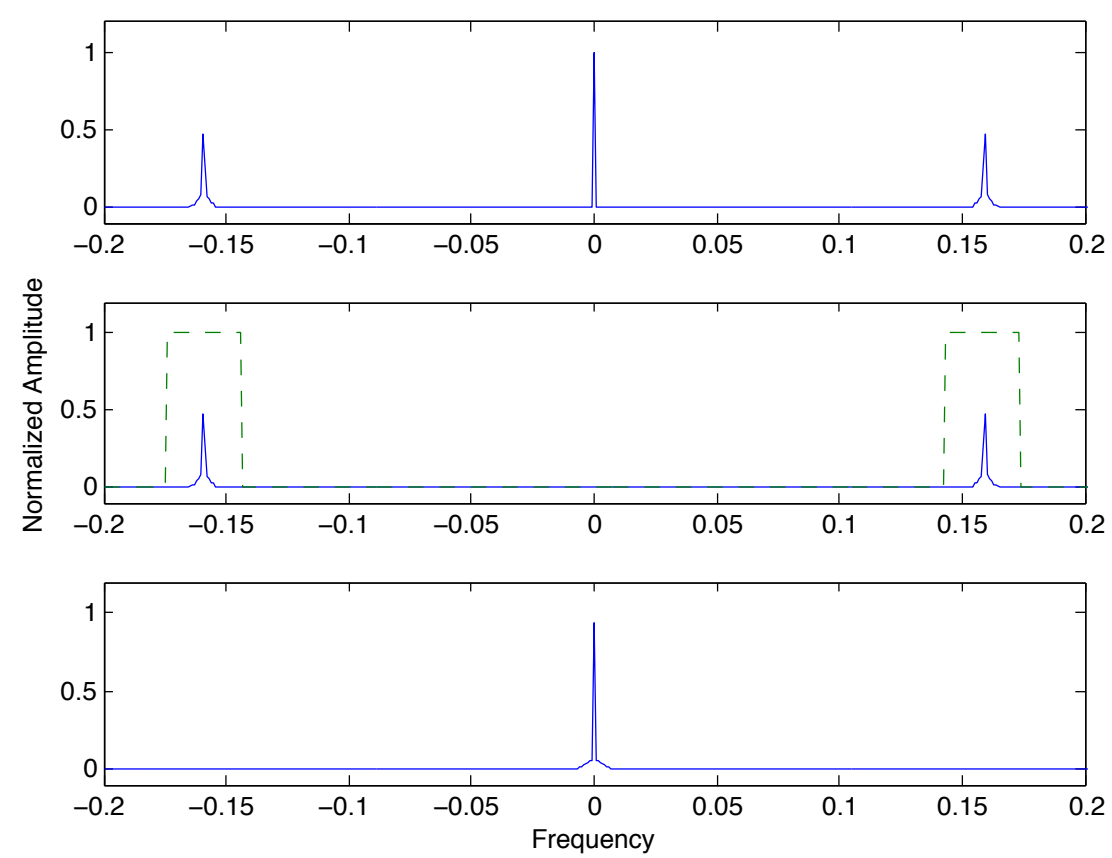

Figure 4.4: Fourier filtering of the modulated interference pattern from the Michelson interferometer.

The wavefront shape is then obtained by from the phase angle $\Phi$ of $u$

$$
\Phi=\tan ^{-1}\left\{\frac{\operatorname{Im}(u)}{\operatorname{Re}(u)}\right\}=k(2 \Delta L-2 \delta) .
$$

Similar to the analysis of the shearing interferograms, we unwrap the phase to 
ensure there are no phase jumps larger than $\pi$ between adjacent pixels, yielding the shape of the physical phase front.


Figure 4.5: A simulated wavefront with and without a deformation (top) and the recovered (Fourier filtered) deformation shape (bottom).

The difference between thermally distorted and undistorted wavefronts, denoted by the subscript $d$ and $r$ respectively, gives twice the thermally induced 
wavefront deformation $\delta$

$$
\begin{aligned}
\Phi_{d}-\Phi_{r} & =\tan ^{-1}\left\{\frac{\operatorname{Im}\left(u_{d}\right)}{\operatorname{Re}\left(u_{d}\right)}\right\}-\tan ^{-1}\left\{\frac{\operatorname{Im}\left(u_{r}\right)}{\operatorname{Re}\left(u_{r}\right)}\right\} \\
& =k(2 \Delta L-2 \delta)-k(2 \Delta L) \\
& =2 k \delta .
\end{aligned}
$$

Example wavefronts, with and without a gaussian deformation $f(x)$, are shown in the top graph of Figure 4.5. The example wavefronts were modulated and their Fourier transforms obtained and filtered as described above. The filtered frequency components were then shifted to zero frequency and the wavefronts reconstructed. The reconstructed deformation is calculated by taking the difference between the deformed and undeformed reconstructed wavefronts and shows excellent agreement with the Gaussian function used to generate the deformation.

\subsection{Shearing Measurement Validation}

To validate the results of the shearing interferometer, the thermal deformation of the test mirror was independently measured using a white-light Michelson interferometer, whose arms measure approximately $10 \mathrm{~cm}$ in length. This interferometer consists of a red light emitting diode whose center wavelength is $623 \pm 3 \mathrm{~nm}$, a pellicle beam splitter and gold coated end mirrors. Figure 4.6 shows a photograph of the Michelson interferometer (yellow dashed line), the pump laser beam (blue) and the probe beam (red); the pump laser heats and deforms the front surface of the test optic which is measured by both the probe laser which goes to the shearing interferometer and the Michelson interferometer which provides a means of calibrating the probe beam measurement. Interferograms from the Michelson interferometer were captured as JPEG images using the same commercially available webcam as for the shearing interferograms. 


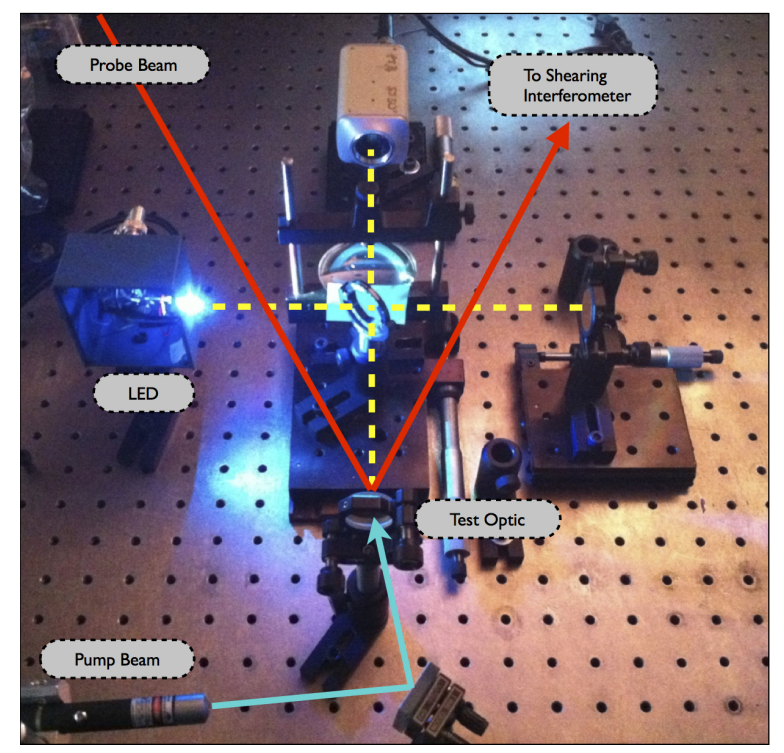

Figure 4.6: A photograph of the Michelson interferometer used in this experiment.

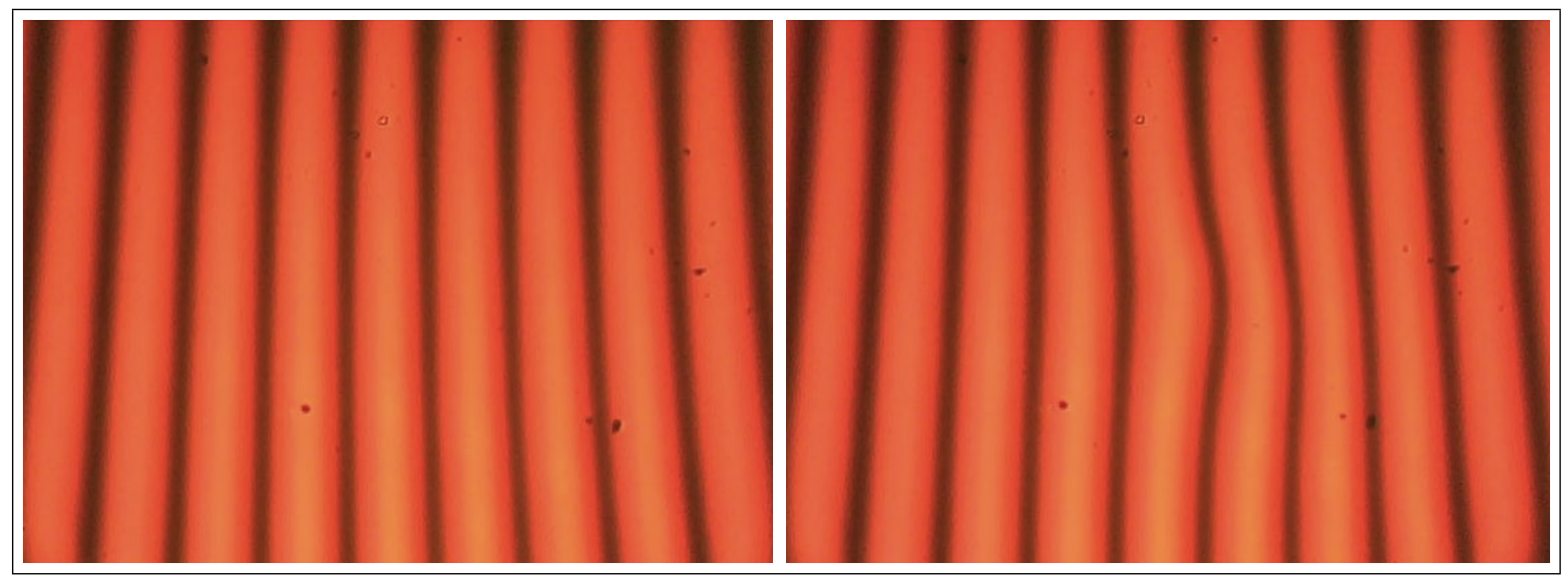

Figure 4.7: An image of the recorded interference pattern due to the undeformed test optic (left) and the thermo-elastically deformed test optic (right).

The reference mirror of the interferometer was tilted to produce fringes on the interference pattern, which was recorded by the camera both with and without the test mirror being subjected to the pump beam that produced the thermal deformation, as shown in Figure 4.7. 


\section{CHAPTER 5}

\section{SHEARING INTERFEROMETER DATA EXTRACTION AND CALIBRATION}

The previous chapter outlined the methods and theoretical groundwork needed to analyze interferograms containing information about the thermo-elastic deformation of a test optic. This chapter will focus on the numerical and

computational methods using MATLAB in conjunction with data collected from the experimental apparatus. This chapter will demonstrate the major relevant parts of the data analysis algorithm.

\subsection{Experimental Details}

In this experiment, the pump helium-neon (HeNe) laser light, polarized at $45^{\circ}$, passes through a Farady isolator to prevent any reflected beams from causing destabilizing feedback into the laser. The beam is then focused through a $5 \mu \mathrm{m}$ pinhole by a $50 \times$ microscope objective lens, shown in Figure 5.1, to clean the spatial mode of the laser. Several steering mirrors then direct the probe beam to the front face of the gold-coated test optic. The optical path length from the pinhole to the test mirror is $168 \mathrm{~cm}$. Several more steering mirrors direct the light an additional $125 \mathrm{~cm}$ to the BBO crystal, resulting in an image magnification of $M$ of $(125+168) / 168=1.74 \times$ from the test mirror to the BBO.

We place a thin, $250 \mu \mathrm{m}$ wire in the beam path just in front of the BBO as is shown in FIgure 5.4. The BBO, whose eigenpolarizations are in the vertical and horizontal directions, has a clear aperture of 12x8 mm and displaces one principle 
polarization state by approximately $835 \mu \mathrm{m}$. We measure the width of the wire's shadow to calibrate the spatial scale of the interferogram and measure the separation of the orthogonally polarized shadows of the pin to empirically determine the shear. A half wave plate oriented at $22.5^{\circ}$ vertical (shown in Figure 5.3) placed after the $\mathrm{BBO}$ rotates both principle polarizations states by $45^{\circ}$.

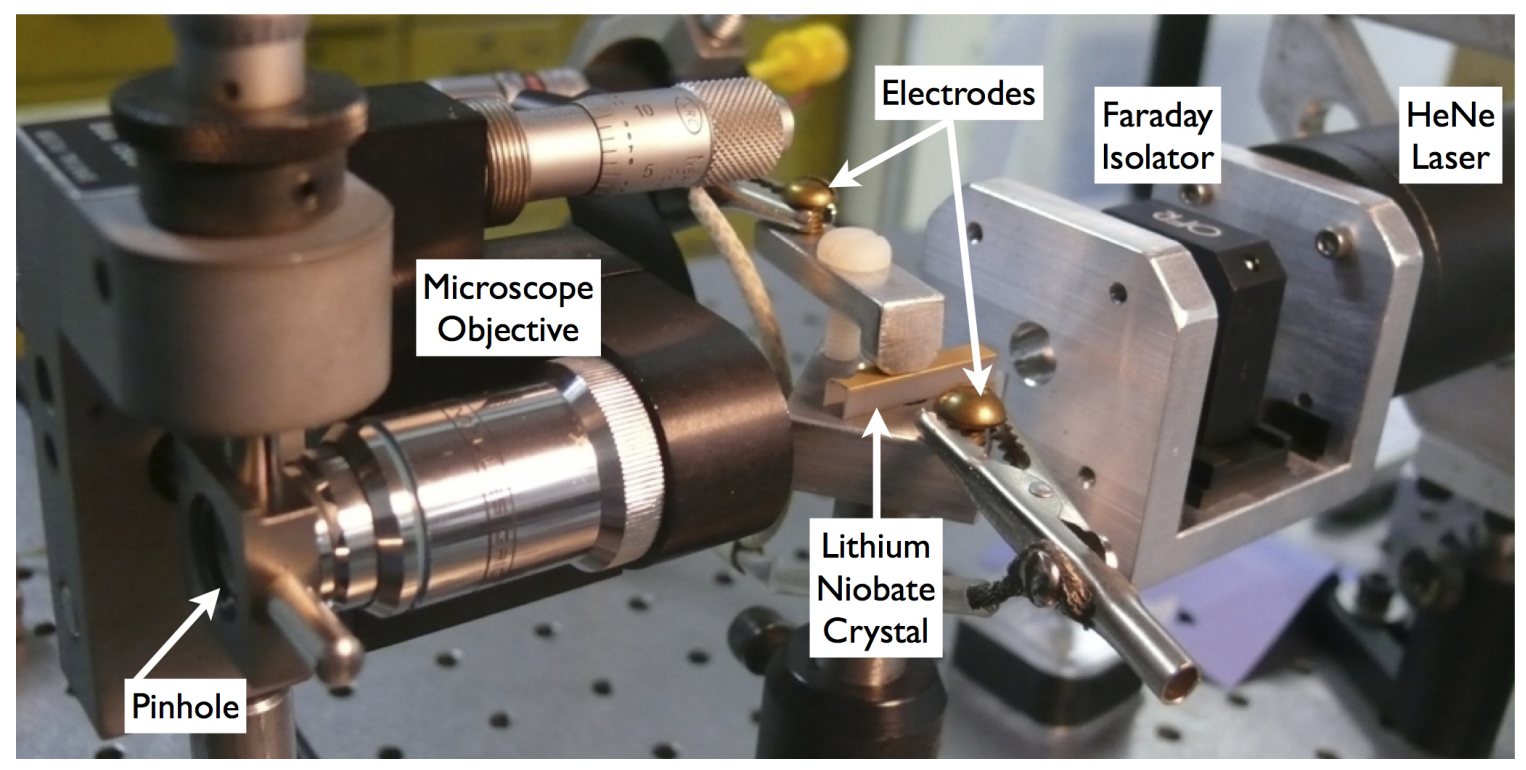

Figure 5.1: A photograph of the HeNe laser, Faraday isolator, electrooptic modulator (consisting of electrodes and a crystal of Lithium Niobate), microscope objective and pinhole used in this experiment.

The light passes through a polarizing beam splitter cube (Figure 5.2) while a $75.0 \mathrm{~mm}$ focal length lens images the front of the $\mathrm{BBO}$ crystal onto the imaging sensor with a magnification of $-0.45 \times$ for a net magnification of the test mirror to onto the imaging sensor of $-0.79 \times$.

The $\mathrm{BBO}$ was placed in ball-bearing mount to allow for easy rotation between the two orthogonal crystal orientations. To ensure the crystal rotation did not introduce unwanted translation of the beam, the shadow of the wire placed in front of the crystal in the field of view of the camera is observed to remain fixed (for the 
ordinary wave), although a small amount of translation of the beam relative to the shear could be tolerated, since the shear determines the resolution of the wavefront reconstruction.

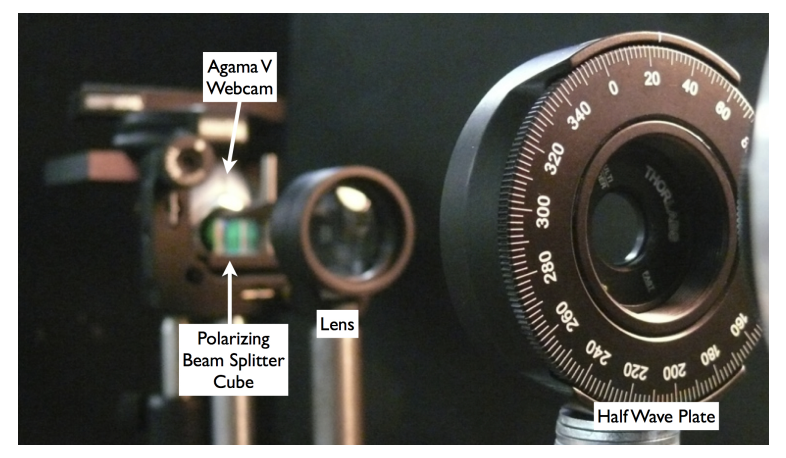

Figure 5.2: A photograph of the half wave plate, lens and polarizing beam splitter used in this experiment.

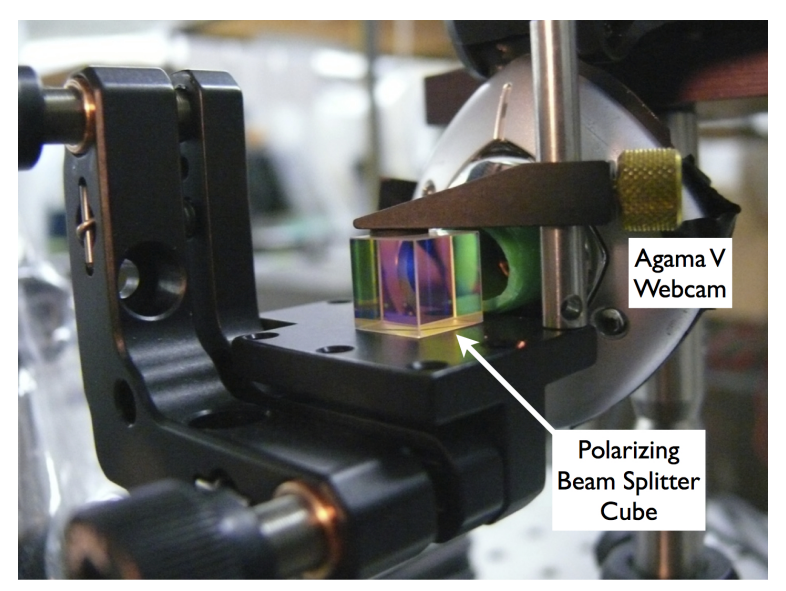

Figure 5.3: A photograph of the Polarizing beam splitter and webcam used in this experiment.

The violet pump laser beam $(\lambda=405 \mathrm{~nm})$ illuminates the back of the gold test mirror at near normal incidence and produces $78.9 \mathrm{~mW}$ of power, with 19.7 $\mathrm{mW}$ reflected from the mirror. With negligible transmission, this gives $59.2 \mathrm{~mW}$ of absorbed power available to cause thermal deformation of the test optic. The pump laser has a Gaussian beam profile with a Gaussian radius of $0.95 \mathrm{~mm}$. 


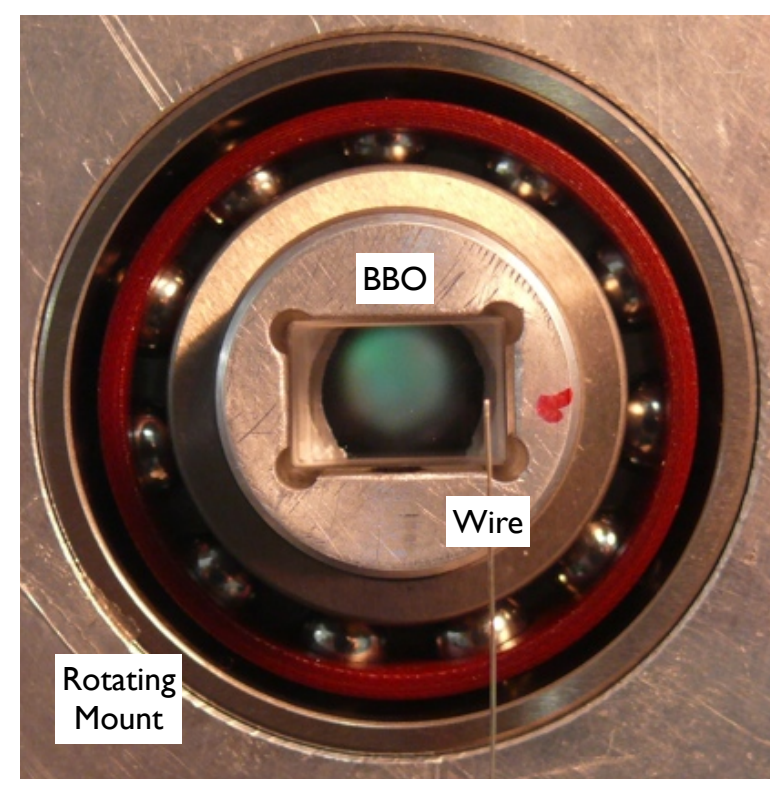

Figure 5.4: A photograph of the BBO in its rotating mount and the wire used to calibrate the spatial scale of the interferogram.

A photograph of the entire bench top experiment is shown in Figure 5.5. The red dashed line in Figure 5.5 denotes the path of the HeNe probe laser beam, the blue dashed line indicates the path of the violet pump laser, and the yellow lines indicate the optical paths in the Michelson interferometer.

\subsection{Calculation and Measurement of Shear}

The crystallographic axes for the particular crystal used in this experiment are cut such that the principle axes $x^{\prime}$ and $z$ are rotated by $\theta=28^{\circ}$ from the surface normal. At the wavelength of the probe HeNe laser beam, $\lambda=632.8 \mathrm{~nm}$, Beta Barium Borate has ordinary and extraordinary indices of refraction $n_{0}=1.6673$ and $n_{e}=1.5500[19]$. Using these details, we are able to calculate the walk-off angle $\rho$ 
between the deviated and undeviated beams in the crystal

$$
\begin{aligned}
\rho & =\cos ^{-1}\left(\frac{\left[\frac{\cos ^{2} \theta}{n_{o}^{2}}+\frac{\sin ^{2} \theta}{n_{e}^{2}}\right]}{\sqrt{\frac{\cos ^{2} \theta}{n_{o}^{4}}+\frac{\sin ^{2} \theta}{n_{e}^{4}}}}\right) \\
& =\cos ^{-1}\left(\frac{\left[\frac{\cos ^{2}\left(28^{\circ}\right)}{(1.6673)^{2}}+\frac{\sin ^{2}\left(28^{\circ}\right)}{(1.5500)^{2}}\right]}{\sqrt{\frac{\cos ^{2}\left(28^{\circ}\right)}{(1.6673)^{4}}+\frac{\sin ^{2}\left(28^{\circ}\right)}{(1.5500)^{4}}}}\right) \\
& =3.6^{\circ} .
\end{aligned}
$$

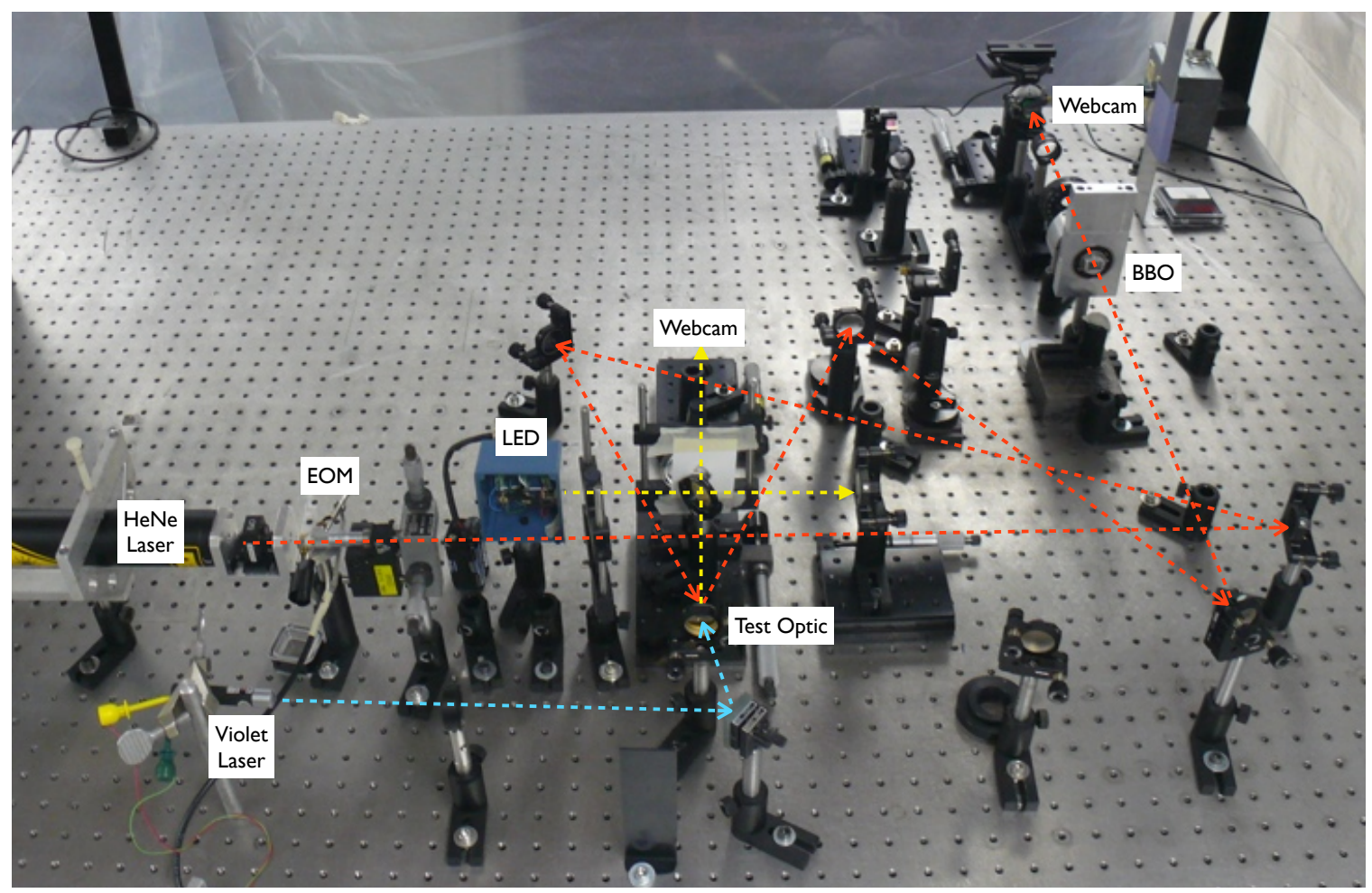

Figure 5.5: A photograph of the entire experiment and all it's components. 
After traversing the length of the crystal $L=13 \mathrm{~mm}$, the two beams are laterally separated by the amount of shear

$$
\begin{aligned}
s & =L \tan (\rho) \\
& =(13 \mathrm{~mm}) \tan \left(3.6^{\circ}\right) \\
& =820 \mu \mathrm{m} .
\end{aligned}
$$

Experimental verification of the above calculations was conducted by placing a $250 \mu \mathrm{m}$ diameter wire just in front of the BBO and the distance between the two shadows (one for each polarization component) was measured. The empirically measured shear of $835 \mu \mathrm{m}$ agrees to within $2 \%$ of the calculated result presented above and is insignificant compared to the uncertainty in the length of the crystal.

Since the two beams experience different optical path lengths there is some static birefringence $\left(\Gamma_{0}\right)$ due to traversing the crystal. However, this static birefringence is common to both the deformed and undeformed interferograms and is of no concern since it cancels out in the analysis.

\subsection{Increasing the Dynamic Range of Shearing Interferograms}

The wavefront deformation is obtained by measuring each component $(x$ and $y$ ) of the distorted and undistorted wavefronts using data obtained from the shearing interferometer. The data from the shearing interferometer consist of a 30 second video of the modulated interferogram collected by a consumer-grade 8-bit color webcam (Agama-V) with a frame-rate of 15 frames per second, giving a Nyquist frequency of $7.5 \mathrm{~Hz}$.

In order for Matlab to properly interpret the video file, and extract relevant video information such as frame rate, screen size and color channels, it is converted into a multimedia reader object using the mmreader command and assigned a 
variable name

$$
\mathrm{MM}=\operatorname{mmreader}(\text { VideoFile })
$$

This multimedia reader object is converted into frame-by-frame arrays, one of

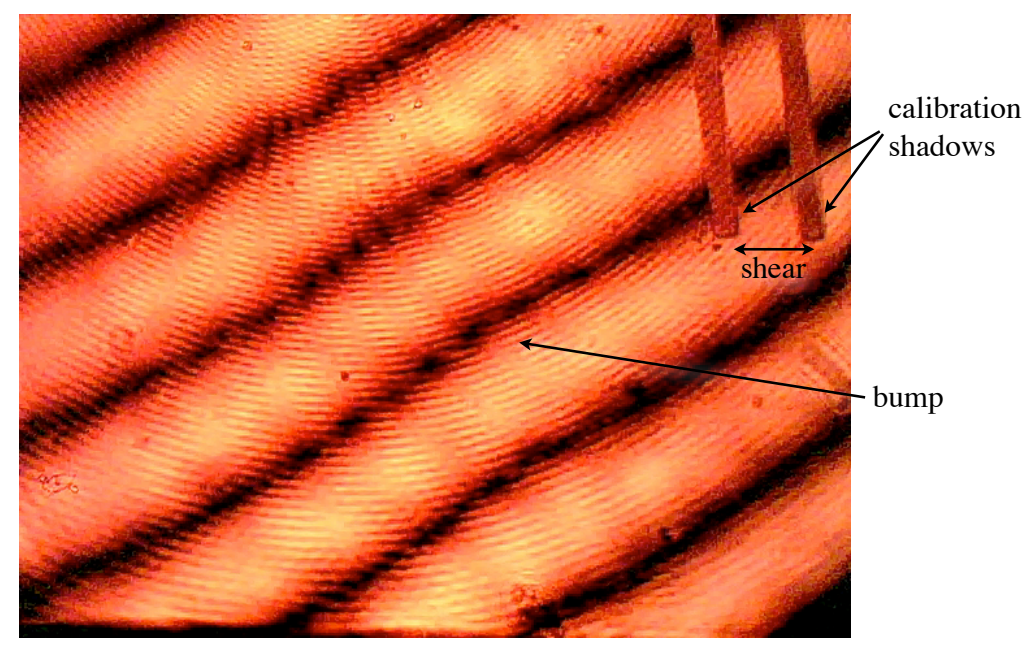

Figure 5.6: An interferogram from the shearing interferometer showing horizontal shear.

which is shown in Figure 5.6

$$
\text { image }=\operatorname{read}(\text { MM, frame })
$$

and each color channel is assigned a unique variable

$$
\begin{aligned}
& \operatorname{Mred}(\text { frame },:,:)=\operatorname{double}(\text { image }(:,:, 1)) \\
& \operatorname{Mgreen}(\text { frame },:,:)=\text { double }(\text { image }(:,:, 2)) \\
& \text { Mblue }(\text { frame },:,:)=\operatorname{double}(\text { image }(:,:, 3)),
\end{aligned}
$$

where the colon operator denotes all the elements in the rows of the array. The effective dynamic range of the webcam is increased by stitching together the data from the red and green channels of each pixel. The red channel is most sensitive to 
the $632.8 \mathrm{~nm}$ light and for our illumination is often saturated at points with constructive interference. The green channel is less sensitive to the $632.8 \mathrm{~nm}$ light, and does not saturate, but fails to register non-zero values for many points with destructive interference, as shown in Figure 5.7.

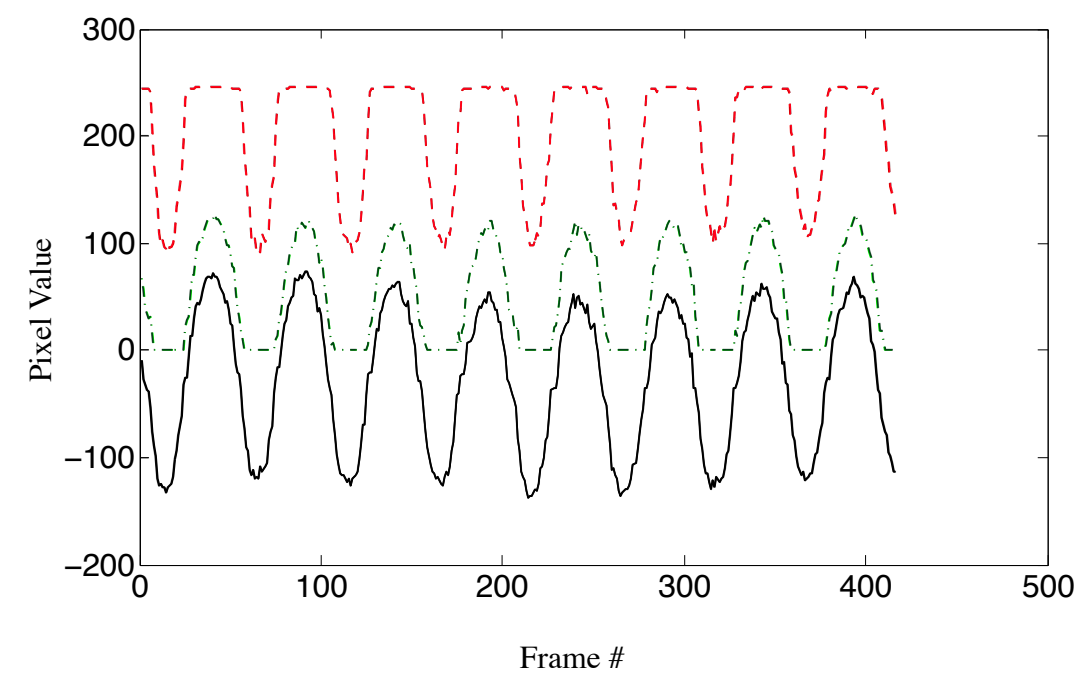

Figure 5.7: Red (upper dashed curve) and green (lower dashed curve) values for a representative pixel as a function of time together with the composite value (solid line) formed by stitching them together after appropriate scaling.

We stitch the red and green channels together to get a composite data channel by choosing a threshold appropriate for the illumination level such that if the green pixel value exceeds this threshold the green data is considered more reliable (as the red would be saturated) and below this threshold the red data is considered more reliable, since the the green data would be near the noise floor. For our illumination, the threshold value was chosen to be 32 (out of 255 for our 8-bit sensor).

We then take the frame-to-frame difference of each data channel so that it is the derivative of the data being stitched together avoiding discontinuities in the composite data. The $\operatorname{diff}()$ command calculates the difference between adjacent 
elements in an array, equivalent to taking a finite derivative

$$
\begin{aligned}
& \text { DiffRed }=\operatorname{diff}(\text { Mred }) \\
& \text { DiffGreen }=\operatorname{diff(Mgreen}) .
\end{aligned}
$$

We empirically determine the appropriate scale factor for the derivative of the green data to compensate for the amplitude difference between the red and green values. The scale factor is determined by comparing the frame-to-frame difference of the data in the red channel to the frame-to-frame difference of the data in the green channel for frames where the data in the green channel crosses our chosen threshold.

To obtain the necessary scale factor, we fit a line to the red and green channels using the polyfit $(\mathrm{X}, \mathrm{Y}, \mathrm{N})$ function, which fits a polynomial of order $\mathrm{N}$ to the function $\mathrm{Y}(\mathrm{X})$, in our case $\mathrm{N}=1$ for a line

$$
\text { Coeffs }=\text { polyfit(DiffRed, DiffGreen, } 1)
$$

The scale factor is the first order coefficient returned by the polyfit() function, that is, the slope $\mathrm{m}$ of the best fit line between these two data sets. The derivative of the green data channel is then scaled by this factor, and replaces the derivative of the red data for regions where the green values exceed our chosen threshold.

$$
\mathrm{dM}=\mathrm{m} . * \text { DiffRed. } *(\text { Mgreen }<32)+\text { DiffGreen. } *(\text { Mgreen }>=32),
$$

where the $* *$ operator denotes term-by-term multiplication. The cumulative sum of this composite derivative data channel, equivalent to integrating the processed derivative to recover the processed version of the original function, is then computed

$$
\mathrm{M}=\operatorname{cumsum}(\mathrm{dM})
$$

to give a composite data channel free of discontinuities and kinks at the stitching points. An example of the time series of data for a single pixel is shown in Figure 5.7. 


\subsection{Calculation and measurement of Modulation Depth}

As described in the previous chapter, we modulate the birefringence of the system cosinusoidally using an electrooptic modulator. For our geometry, the crystal length is $L=25 \mathrm{~mm}$ and the electrodes are $d=4 \mathrm{~mm}$ apart. The electrooptic modulator is made of a crystal of Lithium Niobate which has an ordinary index of refraction $n_{o}=2.286$ and an extraordinary index of refraction $n_{e}=2.200$ [33]. The relevant electro-optic tensor components are $r_{13}=9.6 \mathrm{pm} / \mathrm{V}$ and $r_{33}=30.9 \mathrm{pm} / \mathrm{V}$. The half wave voltage $V_{\pi}$ is

$$
\begin{aligned}
V_{\pi} & =\frac{d}{L} \frac{\lambda}{\left[n_{e}^{3} r_{33}-n_{o}^{3} r_{13}\right]} \\
& =\frac{4 \mathrm{~mm}}{25 \mathrm{~mm}} \frac{632 \mathrm{~nm}}{\left[(2.200)^{3}(30.9 \mathrm{pm} / \mathrm{V})-(2.286)^{3}(9.6 \mathrm{pm} / \mathrm{v})\right]} \\
& =470 \mathrm{~V} .
\end{aligned}
$$

Since the webcam used in this design has a frame rate of 15 frames per second, we must drive the EOM at a frequency below $7.5 \mathrm{~Hz}$. We drive our EOM with $150 \mathrm{~V}$ sinusoidal signal at a frequency of $0.3 \mathrm{~Hz}$, well below the Nyquist frequency for our measurement. Since a voltage of $470 \mathrm{~V}$ produces $\pi$ radians of birefringence, and we use $150 \mathrm{~V}$, we expect to obtain approximately 1 radian of birefringence, so the modulation depth $m$ is 1 .

The modulation depth and frequency were determined implicitly from the video data. Ideally, the modulation frequency would be synchronized to the frame rate of the camera such that each cycle of modulation corresponds to exactly six frames of data. This would allow an integer number of cycles of the fundamental, second and third harmonics of the modulation frequency (those used in the analysis) to be measured without spectral leakage in the discrete Fourier transform, 
while minimizing the acquisition time. Because we had no convenient way to synchronize the modulation and acquisition rate, we instead used a long integration time to measure many cycles of modulation and processed a subset of this data that has a near integer number of modulation cycles, thus minimizing spectral leakage.

To measure the modulation depth, we begin by truncating the time series data to a bit less than 450 points so that it contains an integer number of cycles, and then padding the array with zeros to a length of 1024 and take the fast Fourier transform of each pixel $M$ in the time domain

$$
F=f f t(M, 1024)
$$

We determine the complex amplitude of the first, second and third harmonics by zeroing out the DC peak and manually detecting the index of the first harmonic peak (assigned the variable name peakindex).

$$
\begin{aligned}
& \text { A1o }=\operatorname{squeeze}(\mathrm{F}(\text { peakindex }),:,:) \\
& \mathrm{A} 20=\operatorname{squeeze}(\mathrm{F}(2 * \text { peakindex }),:,:) \\
& \text { A3o }=\operatorname{squeeze}(\mathrm{F}(3 * \text { peakindex }),:,:),
\end{aligned}
$$

The squeeze() function reduces the dimension of the first row, so it contains only the value at the peak index. The peaks of the second and third harmonics occur at twice and three times the index of the first harmonic peak.

In order to shift the time origin so that the modulation waveform is purely cosinusoidal, we record the complex Fourier transform amplitude for each pixel and subtract one, two and three times respectively the measured phase angle of the fundamental. This results in a purely real fundamental component - equivalent to time shifting the data so the modulation is cosinusoidal. The mean value of the 
phase angle (modulo $\pi$ ) of the first harmonic is calculated over all the pixels

$$
\text { theta } 1=\operatorname{mean}(\bmod (\operatorname{angle}(\mathrm{A} 1 \mathrm{o}), \mathrm{pi})) .
$$

The phase angle of the second and third harmonics occur at twice and three times the phase angle of the first. The real part of the complex amplitudes yields the amplitudes $A_{1}, A_{2}$ and $A_{3}$

$$
\begin{aligned}
& \text { A1 }=\operatorname{real}(\text { A10 } * \exp (-i * \text { theta } 1) \\
& \text { A2 }=\operatorname{real}(\text { A20 } * \exp (-i * 2 * \text { theta } 1) \\
& \text { A3 }=\operatorname{real}(\text { A3o } * \exp (-i * 3 * \text { theta } 1)
\end{aligned}
$$

As mentioned in the previous chapter, the ratio of the first and third harmonic amplitudes is given by Bessel Functions such that

$$
\frac{A_{1}}{A_{3}}=-\frac{J_{1}(m)}{J_{3}(m)} .
$$

We have determined the necessary amplitudes on the left hand side of the above equation, and must now determine the value of the modulation depth which satisfies the right hand side. Creating a vector of trial values for $m$ between 0 and 3 radians in steps of 0.01 allows us to compute the ratio of the first and third Bessels functions for each of these values

$$
\begin{aligned}
& \text { mtrial }=(0.0: 0.01: 3) \\
& \mathrm{J} 1 \mathrm{~J} 3=\operatorname{abs}(\operatorname{bessel} j(1, \text { mtrial }) / \text { bessel } j(3, \text { mtrial })) \\
& \text { A1A3 }=\operatorname{abs}(\mathrm{A} 1 / \mathrm{A} 3) .
\end{aligned}
$$

Since the ratio of $A_{1}$ to $A_{3}$ can be obtained from the first order coefficient returned from the best fit line between these two data sets

$$
\begin{aligned}
& \text { coeffs = polyfit }(A 1, A 3,1) \\
& \text { a1a3 }=\operatorname{coeffs}(1)
\end{aligned}
$$


The smallest least squared error between the slope and each entry of the J1J3 array is calculated

$$
\text { delta }=(\mathrm{a} 1 \mathrm{a} 3-\mathrm{J} 1 \mathrm{~J} 3) \cdot{ }^{\wedge} 2
$$

and yields the best fit modulation depth of $m=1.08$, which is in agreement with the result $m \approx 1$ obtained using equation 5.2 .

\subsection{Waverfront Reconstruction From Shearing Interferograms}

With the dynamic range of the shearing interferograms boosted to contain unclipped fringes and the modulation depth determined, the deformed and undeformed wavefronts can be reconstructed. Recall from equation 3.38 we calculate the shear from the amplitude of the first and second harmonics as well as the first and second Bessel functions

$$
\begin{aligned}
& \mathrm{xr}=\mathrm{A} 1(:,:) \cdot / \operatorname{bessel} j(1, \mathrm{mf} i t) \\
& \mathrm{yr}=\mathrm{A} 2(:,:) \cdot / \operatorname{bessel} \mathrm{j}(2, \mathrm{mf} i t) \\
& \mathrm{zr}=\mathrm{yu} . / \mathrm{xu}
\end{aligned}
$$

where the inverse tangent of $\mathrm{zr}$ gives the pixel-by-pixel gradient of the undeformed reference wavefront. A similar analysis on the deformed wavefront (from the video containing the deformed interferogram) yields the inverse tangent of $z \mathrm{~d}$, which is the pixel-by-pixel gradient of the deformed wavefront. In order to avoid introducing errors, the difference of arctangents is computed before being unwrapped to determine the gradient of the shear in the direction of the wavefront $\nabla \delta \cdot \vec{s}$

$$
\operatorname{deltaxS}=\operatorname{unwrap}((\operatorname{zd}(:,:)-\operatorname{zr}(:,:)) \cdot /(1+\operatorname{zd}(:,:) . * \operatorname{zr}(:,:))
$$


Dividing by the dimensionless shear $d x / s$, where $d x$ is the size of a pixel results in the gradient of the phase front $\nabla_{x} \delta$

$$
\text { DelxDelta }=\text { deltaS. } *(\mathrm{dx} / \mathrm{s})
$$

which is then related to the height of the deformation via $2 \nabla_{x} h=\nabla_{x} \delta$ and scaled by a factor of $\lambda / 2 \pi$ to obtain the gradient of the height in nanometers. This process is repeated to obtain the gradient in the $y$-direction. The tilt of the mirror is removed and the arrays are shifted by the average lateral displacement. Finally, the surface profile is reconstructed using the algorithm described in [3], to obtain a peak height of the reconstructed surface deformation (prior to accounting for the spatial filtering of the interferometer) is $77 \mathrm{~nm}$.

\subsection{Spatial Filtering and Shearing Interferometer Spatial Response}

The finite difference of points on the calculated surface profile for our shearing distance of $s=835 \mu \mathrm{m}$ is taken at discrete points separated by $d x=9.8 \mu \mathrm{m}$ corresponding to the spatial resolution of our image sensor. We then subtract the mean value and calculate the cumulative sum, scaling the results by a factor of $d x / s$, which reproduces the original surface profile, filtered by the spatial frequency response of our shearing interferometer. This process effectively smooths the reconstructed surface, resulting in a reduction in the measured peak deformation which is plotted in Figure 3.60 as a function of $s / w$, the ratio of the amount of shear to the Gaussian width of the pump beam $w$ which heats and deforms the mirror. For this experiment, $s / w=835 \mu m / 950 \mu m=0.88$, resulting in a measured peak reduction of $12 \%$. Thus we must multiply our measured height by 1.13 to account for spatial filtering. For the functional form for the thermal deformation in Equation $2.19, l_{c}=0.94 w$, thus we have $s / l_{c}=0.93$ so the shear used in this 
experiment is close to an optimum value for sensitivity and resolution.

\subsection{Shearing Measurement Confirmation}

The JPEG images of the deformed and undeformed interferograms are imported into MATLAB and converted into double precision arrays

$$
\begin{aligned}
& \text { UnDeformed = imread ('UnDeformed.jpg' } \\
& \text { Deformed = imread ('Deformed.jpg') } \\
& \text { UnDeformed = double(UnDeformed }(:,:, 1)) \\
& \text { Deformed = double(Deformed }(:,:, 1)),
\end{aligned}
$$

where $(:,:, 1)$ denotes all the elements in the first column, which corresponds to the pixel values of one color of the JPEG image.

The two-dimensional fast Fourier transform of each array is computed

$$
\begin{aligned}
& \text { FTundeformed }=f f t 2(\text { UnDeformed) } \\
& \text { FTdeformed }=\text { fft2(Deformed). }
\end{aligned}
$$

The DC peak is zeroed out and the remaining peaks of the Fourier transform are located

$$
\begin{aligned}
& \text { PeakUndef ormend }=\max (\max (\operatorname{abs}(\text { FTundeformend })) \\
& \text { PeaksDeformed }=\max (\max (\operatorname{abs}(\text { FTdeformed }))) .
\end{aligned}
$$

The nested $\max ()$ functions are necessary return the largest single value in the $2 \mathrm{D}$ array, the index of which is used to center a super Gaussian bandpass filter $(n=8)$ with a bandwidth of 25 spatial frequency units. Each row of the video contains 640 pixels, so one spatial frequency unit corresponds to $2 \pi / 640 \mathrm{rad} /$ pixel. The filters are 
generated

$$
\begin{aligned}
& \text { FilterUndeformed }=\exp \left(-((\text { PeakindexUndeformed }) \cdot / 25) \cdot{ }^{\wedge} 8\right) \\
& \text { FilterDeformed }=\exp (-((\text { PeakindexDeformed }) \cdot / 25) . \wedge 8)
\end{aligned}
$$

and applied to the data

$$
\begin{aligned}
& \text { UndeformedFiltered }=\text { FTundeformed. } * \text { FilterUndef ormed } \\
& \text { DeformedFiltered }=\text { FTdeformed. } * \text { FilterDeformed. }
\end{aligned}
$$

We then use the circshift() command, which shifts the indices of an array, in order to downshift the remaining modulation peak to zero frequency

$$
\begin{aligned}
& \text { UnDeformedDemod = circshift(UndeformedFiltered, } 1 \text { - PeakindexUndeformed) } \\
& \text { DeformedDemod }=\text { circshift(DeformedFiltered, } 1 \text { - PeakindexDeformed) }
\end{aligned}
$$

and take the inverse Fourier transform

$$
\begin{aligned}
& \text { UnDef ormedProcessed = ifft(UnDeformedDemod) } \\
& \text { DeformedProcessed = ifft(DeformedDemod). }
\end{aligned}
$$

Since the tilted surface of one mirror in the Michelson interferometer has phase modulated the interference pattern, we must unwrap the phase angle of the processed signal

$$
\begin{aligned}
& \text { UnDeformedSurface = unwrap(angle(UnDeformedProcessed })) \\
& \text { DeformedSurface = unwrap(angle(DeformedProcessed }))
\end{aligned}
$$

Finally, we can calculate the height of the surface deformation by subtracting the undeformed surface profile from the deformed surface profile. We must also determine the appropriate conversion factor (with a variable name of convert) 
between the measured phase obtained above, and the physical height of the deformation $h$. Since the round trip distance measured by the interferometer is twice the length of the interferometer's arm, the measured deformation is twice as high as the actually deformation. This results in a phase to height scale factor that is reduced to half of its usual value of $\lambda / 2 \pi$, so convert $=\lambda / 4 \pi$. Thus, the appropriately scaled height of the deformation is

$$
\text { SurfaceBump }=\text { convert. } *(\text { DeformedSurface }- \text { UnDeformedSurface }) \text {. }
$$



Table 5.1: A table of Michelson interferograms taken with varying amounts of reference mirror tilt.

This procedure was done for two different amounts of mirror tilt, corresponding to two different modulation frequencies, to produce equivalent 
deformation maps. Additionally, the deformation of the mirror was measured by aligning the reference mirror to eliminate the tilt between the interfering wavefronts giving a uniform spatial profile of the interference pattern. Each of the interference patterns were recorded with and without the thermal deformation, as shown in Table 5.1, and the intensity of each pixel was compared to the maximum and minimum intensities seen as the reference arm length was scanned through a full fringe, so that the phase of the interference term could be deduced.

\subsection{Measured Sensitivity}

The noise floor of this shearing interferometer was determined from the one-dimensional Fourier transform of a central row of pixels and is used as a representative noise spectrum. At the low spatial frequencies associated with a thermal deformation the average surface gradient noise was $5.8 \times 10^{-6} \mathrm{~m} / \mathrm{m}$. Recall the spatial scaling factor from Equation 3.45 which states $d k^{\prime}=2 \pi s / x$, where the shear is $s=835 \mu \mathrm{m}$ and the width of the measured region is $x=6.3 \mathrm{~mm}$, resulting in $d k^{\prime}=0.86 \mathrm{rad}$ for our experiment. The magnification-independent sensitivity to a gradient, shown in Figure 5.8 is then $5.0 \times 10^{-6}\left(d k^{\prime-1}\right)$ allowing the result to be scaled to account for the shear in sensitivity with different optical magnification and/or shear.

\subsection{Reconstruction Results}

The thermal deformation corresponding to the reconstructed wavefront prior to accounting for the spatial filtering of the shearing interferometer is observed to have a peak of $74 \mathrm{~nm}$. When scaled by a factor of 1.13 to account for spatial filtering, the resulting height is $83 \mathrm{~nm}$, and the corresponding surface profile is 
shown in Figure 5.9. From equation 2.19, the magnitude of the deformation was predicted to be $81 \mathrm{~nm}$, within $3 \%$ of our measured value.

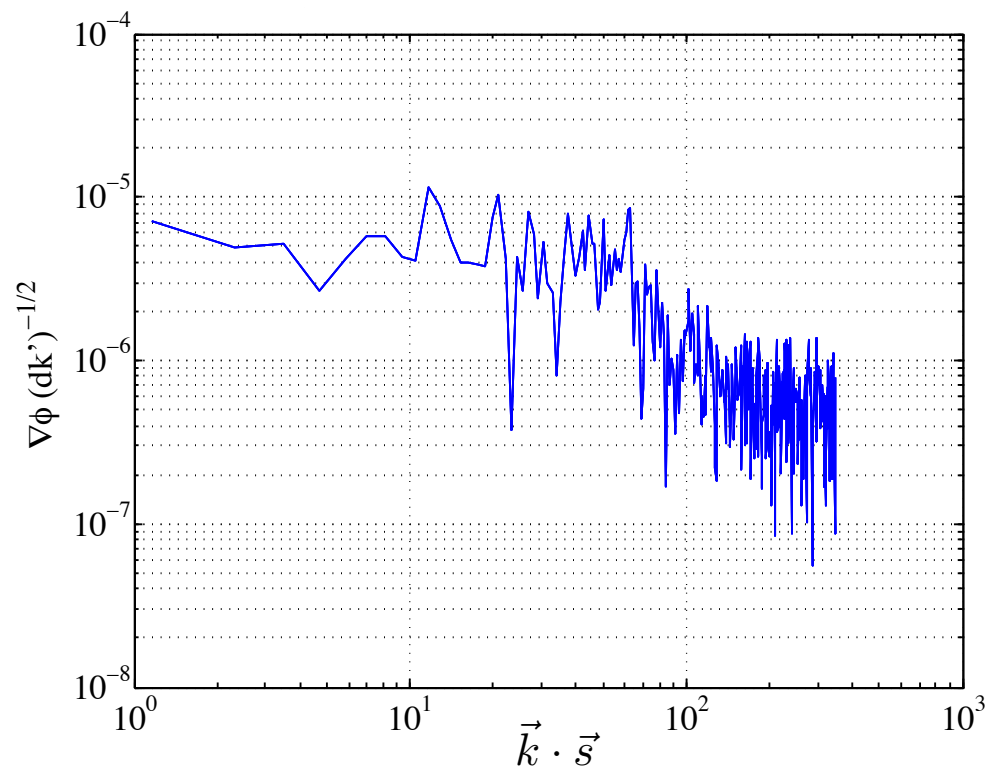

Figure 5.8: Magnification independent sensitivity of the shearing interferometer obtained from a representative row of pixels.

Measurements from the Michelson interferometer were used to validate the results of the shearing interferometer. Measurements using the Michelson interferometer were conducted for two different amounts of mirror tilt, corresponding to 5 and 7 fringes over the field of view to produce equivalent deformation maps. Additionally the deformation of the mirror was measured by aligning the reference mirror to eliminate the tilt between the interfering wavefronts giving a uniform spatial profile of the interference pattern. The interference pattern was recorded with and without the thermal deformation and the intensity of each pixel was compared to the maximum and minimum intensities seen as the reference mirror scanned through a full fringe so that the phase of the interference term could 


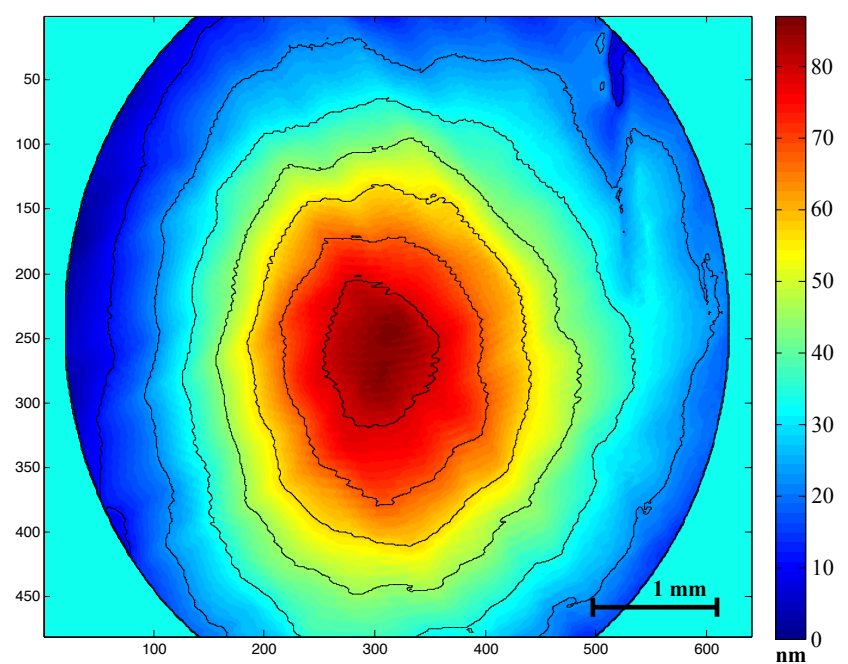

Figure 5.9: Height map of the thermal deformation measurement (in $\mathrm{nm}$ ) from the shearing interferometer after accounting for the spatial frequency response of the interferometer.

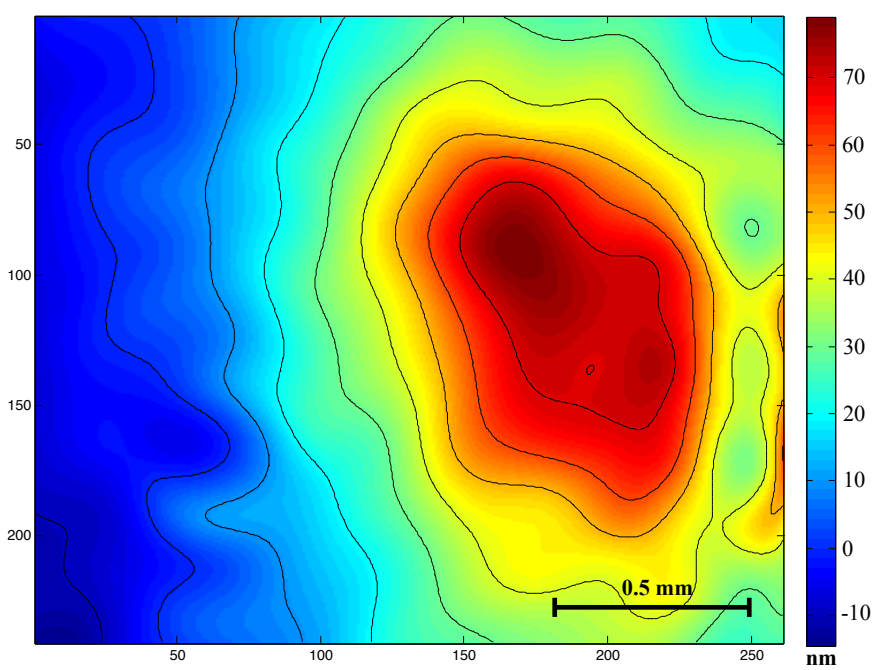

Figure 5.10: Height map of the thermal deformation measurement (in $\mathrm{nm}$ ) from the Michelson interferometer.

be deduced. The wavefronts were reconstructed and the difference was taken to produce a deformation map of the mirror surface, which is shown in Figure 5.10. 
The three measurements of the mirror deformation with the Michelson interferometer yield a magnitude for the surface deformation of $75 \pm 13 \mathrm{~nm}$ consistent with the values found by calculation and from measurements with the shearing interferometer. 


\section{CHAPTER 6}

\section{CONCLUDING REMARKS AND FUTURE DIRECTIONS}

\subsection{Applications}

While relative measurements of the magnitude of an arbitrary deformation are possible, accurately measuring the absolute magnitude of a deformation requires using a shear $s$ that is significantly smaller than the minimum length scale of interest, or requires prior knowledge of the shape of the deformation so that the effect of spatial filtering can be computed independently of the measurement. Thermo-elastic surface deformations of an optic illuminated by a laser beam, such as that described in the work here, meet the latter criteria.

Laser interferometer based gravitational wave detectors have optics that are exposed to high power laser radiation, producing thermal deformations that must be sensed and controlled for operation of the instrument at the design sensitivity [5]. An advanced LIGO intermediate test mass with a radius of curvature of $1934 \mathrm{~m}$ and a beam spot radius of $5.5 \mathrm{~cm}$ has a sagitta measured over the beam spot of $782 \mathrm{~nm}$ [11]. The theoretical sensitivity of the shearing interferometer, given in Equation 3.55 with a Helium-Neon laser and an 8-bit imaging sensor $(\lambda=632.8 \mathrm{~nm}$ and $n=8$ ) allow this to be measured to $0.2 \mathrm{~nm}$, equivalent to a change in the radius of curvature of the mirrors by $0.5 \mathrm{~m}$, well within the required $20 \mathrm{~m}$ accuracy [8].

\subsection{Future Directions}

In principle, the design presented here can be modified in several ways, two of which are presented below. The first alternative design can be implemented with 
only minor changes to the hardware, leaving the software and data analysis scheme largely unchanged; the second alternative design requires more complex changes be made to both the hardware and software.

\subsubsection{Simultaneous Measurement of Gradient in Two Directions}

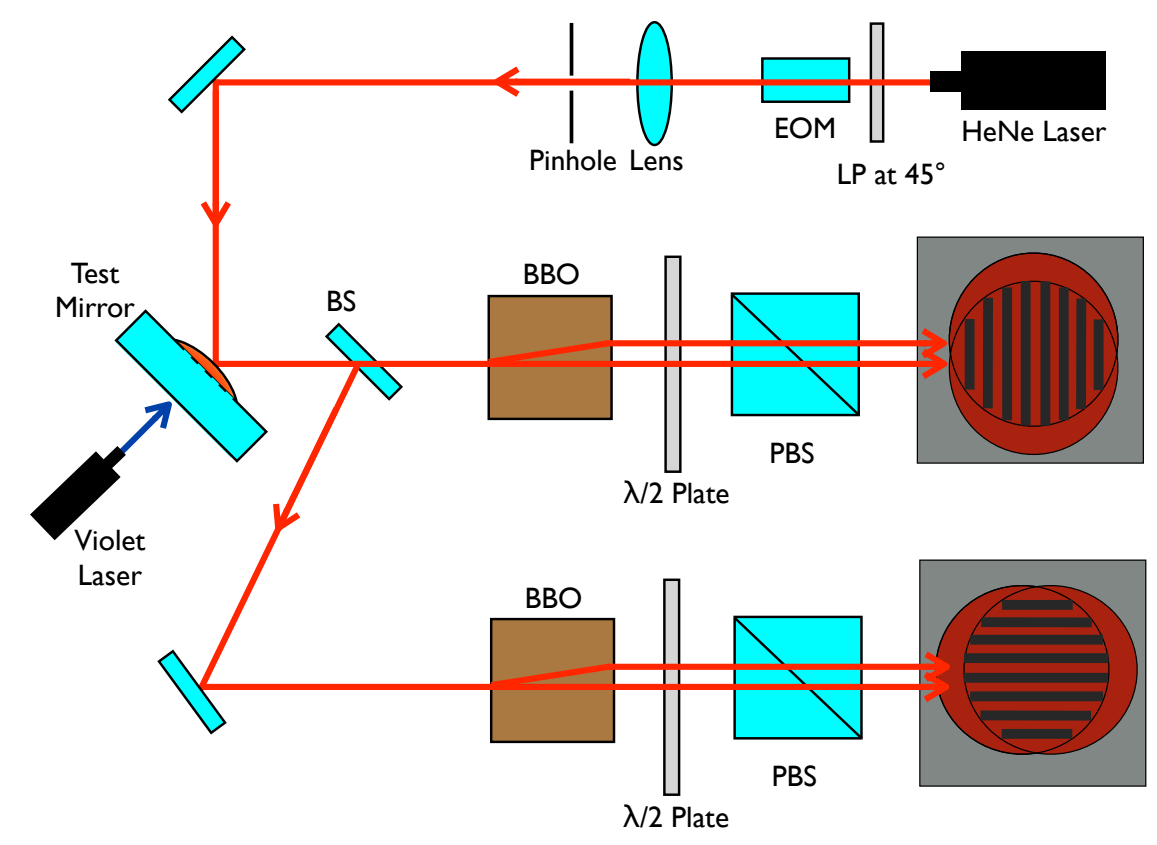

Figure 6.1: An alternative design to the shearing interferometer whereby two arms are used to simultaneously measure the gradient two orthogonal directions.

The first alternative design, shown in Figure 6.1, involves simply adding a second arm to this interferometer. As before, the probe beam passes through a linear polarizer (LP), the electro-optic modulator (EOM), the beam cleaning optics (lens and pinhole) and reflects off the test mirror. The addition of a beam splitter allows simultaneous illumination of a pair of orthogonally oriented BBO crystals, one in each arm of the interferometer. The light in each arm then passes through a half wave plate $(\lambda / 2$ plate) and polarizing beam splitter (PBS) before illuminating an imaging sensor. 
Besides having the ability to measure orthogonal components of the wavefront gradient simultaneously rather than sequentially, this design has the added advantage that the interferogram processing scheme remains entirely identical to that presented in this paper, only the addition of a second data channel is necessary.

\subsubsection{Realtime Measurement of Thermal Deformations}

A design capable of conducting realtime measurements of thermal deformations is shown in Figure 6.2. This design is more complex than either scheme presented thus far and requires modifications to both the software and the hardware. Here, the electro-optic modulator has been removed, but the beam cleaning optics remain. After reflecting off the test optic, the linearly polarized probe beam then passes through a quarter wave plate $(\lambda / 4$ plate), oriented such that the beam is circularly polarized after passing through it. The quarter wave plate is necessary because in this design, the $\mathrm{BBO}$ crystal rotates in a plane perpendicular to the optical axis, so circular polarization ensures there will always be a polarization component along each principle axis of the BBO. The beam then passes through another quarter wave plate which restores linear polarization. As before, the light finally passes through a polarizing beamsplitter before being recorded by the imaging sensor. The EOM in this design has been removed and modulation of the interferogram is instead achieved through the (slow) rotation of the BBO crystal.

The data reduction algorithm must also be adjusted to compensate for changes in the hardware. Since the BBO rotates, the direction of shear also rotates, which must be accounted for during the data processing. As before, a pin placed just in front of the $\mathrm{BBO}$ will cast two shadows, the tips of which can be used to determine the direction of the shear at any one point in time. 


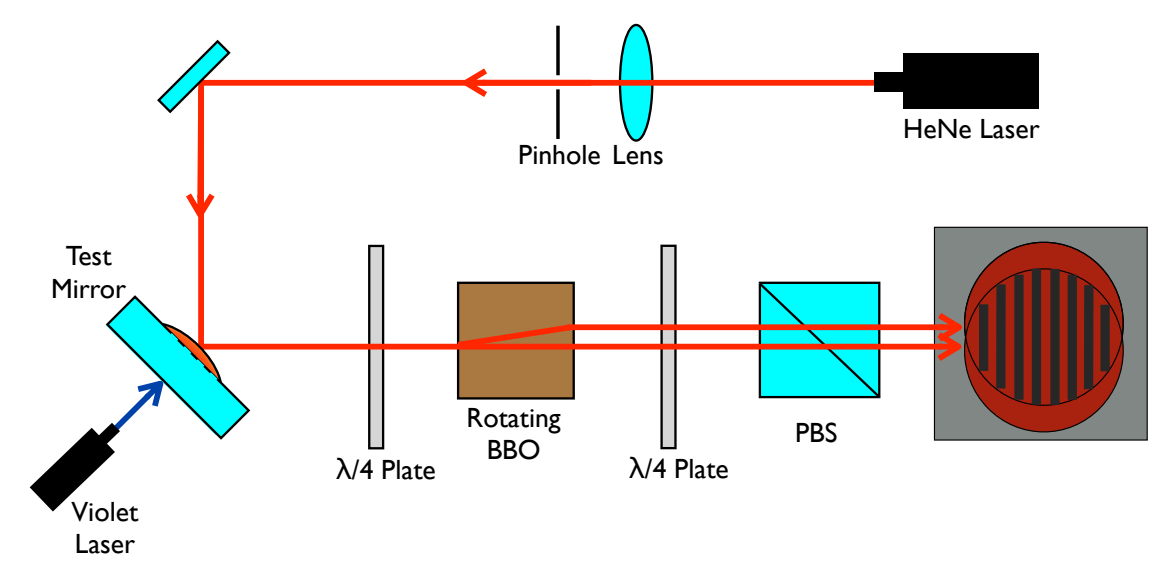

Figure 6.2: A second alternative design to the shearing interferometer where the modulation is achieved by rotating the BBO in a plane perpendicular to the optical axis allowing realtime measurement of thermal deformations.

\subsection{Conclusion}

We have presented a shearing interferometer using polarization control and spatial walk-off in a birefringent crystal to generate shear. This configuration requires no critical alignment and has excellent common-mode noise rejection. We have demonstrated the ability to detect the wavefront distortion produced by thermo-elasetic deformation in an optic heated by absorption of radiation from a laser beam. Our instrument uses post-processed video of modulated interferograms to interpret the wavefront distortion. We leave the development of software to process interferograms in real time as future work.

Our instrument has a sensitivity to surface gradients as small as $5.8 \times 10^{-6}$, which allows a sensitivity of $3.8 \mathrm{~nm}(\lambda / 166)$ of thermo-elastic deformation from heating by a Gaussian laser beam. We have shown the sensitivity is optimized when the size of the shear is comparable to the characteristic length scale of the distortion being probed, and that this optimized displacement sensitivity is scale invariant. We have shown how the effect of spatial filtering by the shearing interferometer can 
be calculated for a known distortion profile, and we have computed the magnitude of this effect for a thermo-elastic deformation of a mirror heated by a Gaussian beam as a function of the amount of shear. Our measurement of the thermo-elastic deformation of a mirror is consistent with an analytical model and with an independent measurement using a white-light Michelson interferometer. 


\section{BIBLIOGRAPHY}

[1] B. Abbott et al. Ligo: The Laser Interferometer Gravitational-Wave Observatory. Reports on Progress In Physics, 72(076901):25-51, Dec 2009.

[2] Abramowitz and Stegun, editors. Handbook of mathematical functions : with formulas, graphs, and mathematical tables. Dover Publications, New York, 1970. page 80, 4.4.36: http://people.math.sfu.ca/ cbm/aands/page_80.htm.

[3] A. Agrawal, R. Chellappa, and R. Raskar. An algebraic approach to surface reconstruction from gradient fields. In Computer Vision, 2005. ICCV 2005. Tenth IEEE International Conference on, volume 1, pages $174-181$ Vol. 1, oct. 2005 .

[4] S. Ballmer, V. Frolov, R. Lawrence, W. Kells, G. M. K. Mason, D. Ottaway, M. Smith, C. Vorvick, P. Willems, and M. Zucker. Initial LIGO Thermal Compensation System. LIGO- T050064-00-R, 2009.

[5] S. Ballmer, V. Frolov, R. Lawrence, W. Kells, G. Moreno, K. Mason, D. Ottaway, M. Smith, C. Vorvick, P. Willems, and M. Zucker. Thermal compensation system description, 2005. [LIGO- T050064-00-R].

[6] E. D. Black. An introduction to Pound-Drever-Hall laser frequency stabilization. American Journal of Physics, 69:79-87, January 2001.

[7] A. F. Brooks. Hartmann Wavefront Sensors for Advanced Gravitational Wave Interferometers. PhD thesis, University of Adelaide, 2007.

[8] M. Flanigan and G. Billingsley. Advanced ligo input test mass (itm), 2008. LIGO-E080511-V3-D.

[9] G. R. Fowels. Introduction to Modern Optics. Dover Publications, Inc., New York, second edition, 1975.

[10] D. C. Ghiglia and M. D. Pritt. Two-dimensional phase unwrapping: theory, algorithms, and software. Wiley, 1998. matlab code posted by Bruce Spottiswoode.

[11] G. Harry. Advanced ligo test masses and core optics, 2010. LIGO-G1000098.

[12] P. Hello and J. Y. Vinet. Analytical models of thermal aberrations in massive mirrors heated by high power laser beams. French Journal of Physics, 51:1267-1282, June 1990. 
[13] P. Hello and J. Y. Vinet. Analytical models of transient thermoelastic deformations of mirrors heated by high power cw laser beams. French Journal of Physics, 51:2243-2261, October 1990.

[14] M. P. Kothiyal and C. Delisle. Shearing interferometer for phase shifting interferometry with polarization phase shifter. Appl. Opt., 24(24):4439-4442, Dec 1985.

[15] R. C. Lawrence. Active Wavefront Correction in Laser Interferometric Gravitational Wave Detectors. PhD thesis, Massachusetts Institute of Technology, 2003.

[16] LIGO Scientific Collaboration. LIGO laser interferometer gravitational-waveobservatory, 2012. [Online; accessed 26-August-2012].

[17] P. P. Lu, A. L. Bullington, P. Beyersdorf, S. Traeger, J. Mansell, R. Beausoleil, E. K. Gustafson, R. L. Byer, and M. M. Fejer. Wavefront distortion of the reflected and diffracted beams produced by the thermoelastic deformation of a diffraction grating heated by a gaussian laser beam. J. Opt. Soc. Am. A, 24(3):659-668, Mar 2007.

[18] V. Murukeshan, O. L. Seng, and A. Asundi. Polarization phase shifting shearography for optical metrological applications. Optics $\mathbb{E}$ Laser Technology, 30(8):527 - 531, 1998.

[19] D. N. Nikogosyan. Beta Barium Borate (BBO) A Review of Its Properties and Applications. Applied Physics A, 52:541-545, March 1991.

[20] F. L. Pedrotti, L. S. Pedrotti, and L. M. Pedrotti. Introduction to Optics. Pearson Prentice Hall, third edition, 2007.

[21] V. Quetschke. LIGO - Coherent optical length measurement with $10^{-18} \mathrm{~m}$ accuracy. In Chemistry and Physics Publications, volume 44, July 2007.

[22] V. Rosso, L. Zhang, F. Michel, Y. Renotte, Y. Lion, and A.-M. Habraken. Out-of-plane displacement derivative measurement: comparison of results obtained by a shearographic interferometer using the separation of the polarization states and the finite element method. In P. Mathieu, editor, Proceedings of Photonics North Conference 2006, volume 6343, page 634327. SPIE, 2006.

[23] M. Servin, M. Cywiak, and A. Davila. Extreme shearing interferometry: Theoretical limits with practical consequences. Opt. Express, 15(26):17805-17818, Dec 2007. 
[24] J. R. Smith. The Path to the Enhanced and Advanced LIGO Gravitational-Wave Detectors. Classical Quantum Gravity, 26:1-10, June 2009.

[25] A. L. Team. Advanced LIGO Reference Design. LIGO-M060056-v1, 2009.

[26] D. Voelz. Computational Fourier Optics A MATLAB Tutorial. SPIE Press, 2011.

[27] J. M. Weisberg and J. Taylor. The relativistic binary pulsar b1913 + 16: thirty years of observations and analysis. In Binary Radio Pulsars, volume 328, pages 25-31, 1991.

[28] Wikipedia. Gravitational wave - wikipedia, the free encyclopedia, 2012. [Online; accessed 26-August-2012].

[29] P. Willems, A. Brooks, M. Smith, and K. Mailand. Advanced LIGO Thermal Compensation System Preliminary Design. LIGO-T0900304-v2, 2009.

[30] W. Winkler, K. Danzmann, A. Rüdiger, and R. Schilling. Heating by optical absorption and performance of interferometric gravitational-wave detectors. Physical Review A, 44:7022-7036, December 2001.

[31] S. Wise, V. Quetschke, A. J. Deshpande, G. Mueller, D. H. Reitze, D. B. Tanner, B. F. Whiting, Y. Chen, A. Tünnermann, E. Kley, and T. Clausnitzer. Phase effects in the diffraction of light: Beyond the grating equation. Phys. Rev. Lett., 95(1):013901, Jun 2005.

[32] H. Yamamoto. Mode matching and diffraction loss of FP cavity with thermal deformations and TCS . LIGO-T0900306-v6, 2010.

[33] A. Yariv and P. Yeh. Optical Waves in Crystals, Propagation and Control of Laser Radiation. Wiley \& Sons, Inc., 2003.

[34] L. Zhu, Tuan, and V. K. Kirillovski. Computerized shearing interferometer. J. Opt. Technol., 75(3):156-160, Mar 2008. 